\title{
Response Calls Evoked by Playback of Natural 50-kHz Ultrasonic Vocalizations in Rats
}

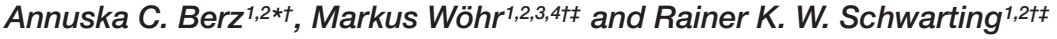 \\ ${ }^{1}$ Behavioral Neuroscience, Experimental and Biological Psychology, Faculty of Psychology, Philipps-University Marburg, \\ Marburg, Germany, ${ }^{2}$ Center for Mind, Brain and Behavior, Philipps-University Marburg, Marburg, Germany, ${ }^{3}$ Research Unit \\ Brain and Cognition, Laboratory of Biological Psychology, Social and Affective Neuroscience Research Group, Faculty \\ of Psychology and Educational Sciences, KU Leuven, Leuven, Belgium, ${ }^{4}$ Leuven Brain Institute, KU Leuven, Leuven, \\ Belgium
}

\section{OPEN ACCESS}

Edited by:

Susanna Pietropaolo,

UMR 5287 Institut de Neurosciences Cognitives et Intégratives d'Aquitaine (INCIA), France

Reviewed by: Stefan Brudzynski, Brock University, Canada

Sergio Marcello Pellis, University of Lethbridge, Canada

${ }^{*}$ Correspondence: Annuska C. Berz Annuska.berz@staff.uni-marburg.de

${ }^{\text {tORCID: }}$

Annuska C. Berz orcid.org/0000-0002-5522-0188

Markus Wöhr orcid.org/0000-0001-6986-5684

Rainer K. W. Schwarting orcid.org/0000-0002-4686-3974

₹These authors share last authorship

Specialty section:

This article was submitted to Individual and Social Behaviors, a section of the journal

Frontiers in Behavioral Neuroscience

Received: 09 November 2021

Accepted: 24 December 2021

Published: 14 January 2022

Citation:

Berz AC, Wöhr M and Schwarting RKW (2022) Response Calls Evoked by Playback of Natural $50-\mathrm{kHz}$ Ultrasonic Vocalizations in Rats.

Front. Behav. Neurosci. 15:812142. doi: 10.3389/fnbeh.2021.812142
Rats are highly social animals known to communicate with ultrasonic vocalizations (USV) of different frequencies. Calls around $50 \mathrm{kHz}$ are thought to represent a positive affective state, whereas calls around $22 \mathrm{kHz}$ are believed to serve as alarm or distress calls. During playback of natural $50-\mathrm{kHz}$ USV, rats show a reliable and strong social approach response toward the sound source. While this response has been studied in great detail in numerous publications, little is known about the emission of USV in response to natural $50-\mathrm{kHz}$ USV playback. To close this gap, we capitalized on three data sets previously obtained and analyzed USV evoked by natural 50-kHz USV playback in male juvenile rats. We compared different rat stocks, namely Wistar (WI) and Sprague-Dawley (SD) and investigated the pharmacological treatment with the dopaminergic D2 receptor antagonist haloperidol. These response calls were found to vary broadly inter-individually in numbers, mean peak frequencies, durations and frequency modulations. Despite the large variability, the results showed no major differences between experimental conditions regarding call likelihood or call parameters, representing a robust phenomenon. However, most response calls had clearly lower frequencies and were longer than typical $50-\mathrm{kHz}$ calls, i.e., around $30 \mathrm{kHz}$ and lasting generally around $0.3 \mathrm{~s}$. These calls resemble aversive $22-\mathrm{kHz}$ USV of adult rats but were of higher frequencies and shorter durations. Moreover, blockade of dopamine D2 receptors did not substantially affect the emission of response calls suggesting that they are not dependent on the D2 receptor function. Taken together, this study provides a detailed analysis of response calls toward playback of $50-\mathrm{kHz}$ USV in juvenile WI and SD rats. This includes calls representing $50-\mathrm{kHz}$ USV, but mostly calls with lower frequencies that are not clearly categorizable within the so far known two main groups of USV in adult rats. We discuss the possible functions of these response calls addressing their communicative functions like contact or appeasing calls, and whether they may reflect a state of frustration. In future studies, response calls might also serve as a new read-out in rat models for neuropsychiatric disorders, where acoustic communication is impaired, such as autism spectrum disorder.

Keywords: ultrasonic vocalizations, animal communication, playback, stock, strain, haloperidol, Wistar, SpragueDawley 


\section{INTRODUCTION}

Acoustic communication among conspecifics is an important aspect of the social life of many species and often essential for maintaining stable social structures. A characteristic feature of acoustic communication in several species is its reciprocal nature where a signal emitted by the sender frequently evokes the emission of a response signal in the receiver (Seyfarth and Cheney, 2003).

Many rodent species communicate through so-called ultrasonic vocalizations (USV), i.e., within frequencies not audible for humans (Brudzynski, 2010). In juvenile and adult rats, two main types of vocalizations are typically distinguished (Brudzynski, 2013a; Wöhr and Schwarting, 2013). Vocalizations with frequencies around $22 \mathrm{kHz}$ are referred to as aversive or distress calls, presumably representing a negative affective state (Blanchard et al., 1991; Fendt et al., 2018). Vocalizations with frequencies around $50 \mathrm{kHz}$ are thought to represent a positive affective state usually emitted during appetitive situations like play or mating (Knutson et al., 1998; Panksepp, 2005). These appetitive calls are typically characterized by frequencies between 35 and $80 \mathrm{kHz}$ and durations in a range of 10-150 ms (Burgdorf et al., 2008; Wöhr et al., 2008; Takahashi et al., 2010). Often, such $50-\mathrm{kHz}$ USV are categorized and the call categories flat, step, trill, and mixed are commonly differentiated (Kisko et al., 2018). Aversive $22-\mathrm{kHz}$ USV, in contrast, have been defined between frequencies of 18 and $32 \mathrm{kHz}$ (Brudzynski, 2001) and within this frequency range, short $(<300 \mathrm{~ms})$ and long (>300 ms) calls were identified (Brudzynski et al., 1993). Long $22-\mathrm{kHz}$ calls were found to be emitted during situations of external danger, such as during the presence of a predator or during predator odor exposure, and are usually associated with freezing behavior (Blanchard et al., 1991; Fendt et al., 2018; Simmons et al., 2018). Short 22-kHz USV, however, are much more ambiguous and their function has not been identified yet (Brudzynski, 2021). It was suggested that short $22-\mathrm{kHz}$ USV represent internal distress without external influence, like frustration (Taylor et al., 2019). In addition, they were repeatedly reported to occur during drug withdrawal (Ma et al., 2010; Simmons et al., 2018).

The communicative functions of $22-$ and $50-\mathrm{kHz}$ USV can be studied by means of playback experiments (Seffer et al., 2014) and it was shown that they elicit distinct behavioral responses pattern in the receiver (Wöhr et al., 2016). Playback of natural 22-kHz USV usually induces a defensive response, including avoidance behavior and behavioral inhibition (Brudzynski and Chiu, 1995; Fendt et al., 2018). Playback of natural 50-kHz USV, in contrast, evokes social approach behavior toward the sound source (Wöhr and Schwarting, 2007). At the physiological level, playback of 22and $50-\mathrm{kHz}$ USV entail to distinct alterations. While playback of $22-\mathrm{kHz}$ leads to a decrease in heart rate during behavioral inhibition, heart rate is increased during social approach behavior in response to playback of 50-kHz USV (Olszyński et al., 2020). Likewise, distinct brain activation patterns are observed. Playback of $22-\mathrm{kHz}$ USV induces increased activity in the amygdala (Sadananda et al., 2008; Parsana et al., 2012), whereas playback of $50-\mathrm{kHz}$ USV results in an activation of the nucleus accumbens
(Sadananda et al., 2008), where it causes a phasic release of dopamine (Willuhn et al., 2014).

At the behavioral level, the social approach response toward $50-\mathrm{kHz}$ USV playback can be accompanied by the emission of response calls (Wöhr and Schwarting, 2007, 2009; Willadsen et al., 2014; Willuhn et al., 2014; Engelhardt et al., 2017, 2018; Berg et al., 2018, 2021; Kisko et al., 2020; Olszyński et al., $2020,2021)$. Although echoing the reciprocal nature of acoustic communication and repeatedly observed in studies applying the $50-\mathrm{kHz}$ USV playback paradigm, still little is known about such response calls. In previous studies, response calls toward $50-\mathrm{kHz}$ USV were observed in males and females (Berg et al., 2018, 2021), albeit the emission of calls in response to $50-\mathrm{kHz}$ USV playback was found to be more prominent in males than females in one study (Kisko et al., 2020). A developmental study further suggests that age is another relevant factor, with juvenile rats emitting more response calls than adult rats (Wöhr and Schwarting, 2009). Finally, prior experiences (Olszyński et al., 2021) and interindividual differences (Engelhardt et al., 2018) were also reported to play a role. However, the function of response calls remains elusive, which is why we wanted to shed light onto the meaning and the importance of response calls in social situations like the $50-\mathrm{kHz}$ USV playback.

To close this gap, we capitalized on a previously obtained large data set and analyzed USV evoked by natural $50-\mathrm{kHz}$ USV playback in male juvenile rats (Berz et al., 2021). In our previous study, we showed, amongst others, that the social approach response toward $50-\mathrm{kHz}$ calls is a stable phenomenon that occurs in Wistar (WI) and Sprague-Dawley (SD) rats and that it can be modulated by administration of the dopaminergic D2 receptor antagonist haloperidol (Halo; Berz et al., 2021). Here, we present three new data sets from these previous experiments. Data set 1 was comprised of WI rats exposed to $50-\mathrm{kHz}$ USV playback. We analyzed it in an initial attempt to better understand the emission of response calls and to test whether response calls occur specifically in reaction toward $50-\mathrm{kHz}$ USV but not noise and whether stimulus order of $50-\mathrm{kHz}$ USV and noise plays a role. Data set 2 consisted of WI and SD rats and their response calls were compared to see whether there was a difference between the stocks. In the final data set 3 , rats received either Halo or saline (Sal) to investigate whether Halo treatment not only affects social approach behavior but also the emission of response calls toward $50-\mathrm{kHz}$ USV playback. Our comprehensive analysis approach included a detailed investigation of the temporal emission pattern and an examination of acoustic features, focusing on numbers of calls, latencies to start calling, mean peak frequencies, call durations, and frequency modulations.

\section{MATERIALS AND METHODS}

\section{Animals and Housing}

In total, 108 experimentally naïve juvenile male rats around 57 weeks of age (Charles River Laboratories, Sulzfeld, Germany) were analyzed. The sample consisted of 90 Wistar (WI) wildtype rats and 18 Sprague-Dawley (SD) wildtype rats. The animals were kept in a vivarium with a 12-hour light/dark cycle with lights on 
at 7 am and $32-50 \%$ humidity. They were housed in groups of five to six rats in polycarbonate cages (macrolon type IV, size $380 \times 200 \times 590 \mathrm{~mm}$ with high steel covers) where food and water were provided ad libitum. After arrival from the breeder, the animals had seven days to acclimate to the vivarium, followed by a standardized protocol of handling for three consecutive days, each day for $5 \mathrm{~min}$. The procedures had been approved by the ethical committee of the local government (Regierungspräsidium Gießen, Germany, TVA Nr. 6 35-2018).

\section{Overview}

Response calls were analyzed in three data sets. These sets were obtained as part of a recently published study focusing on the habituation of the social approach response to repeated playback of $50-\mathrm{kHz}$ USV (Berz et al., 2021). In this previous study, rats were exposed twice to playback of $50-\mathrm{kHz}$ USV and their behavioral response was quantified, i.e., locomotor activity and approach behavior. Here, we now analyzed response calls evoked by playback of $50-\mathrm{kHz}$ USV that were also recorded in this study. We focused on the emission of response calls during the first playback exposure because preliminary data indicate that call emission decreases with repeated playback presentations similar to social approach behavior (Berz et al., 2021). In the first data set, we analyzed response calls in WI rats $(N=24)$ and tested whether their emission occurs specifically during playback of 50$\mathrm{kHz}$ USV but not noise and whether their emission depends on stimulus order. Rats were weighing $144.25 \pm 1.88 \mathrm{~g}$ (range 128.5$164.5 \mathrm{~g})$. In the second data set, we compared the production of response calls between WI rats $(N=18)$ to that of SD rats $(N=18)$. Rats were weighing $163.47 \pm 2.85 \mathrm{~g}$ (range 138.5-205 g). In the third data set, we studied the role of the dopaminergic system in regulating the emission of response calls and compared response calls emitted by WI rats systemically treated with the dopaminergic D2 receptor antagonist Halo $(N=24)$ and saline treated controls $(N=24)$. Rats were weighing $189.57 \pm 2.95 \mathrm{~g}$ (range 147.5-233 g).

\section{Drug Treatment}

In the third data set, rats received the dopaminergic D2 receptor antagonist Halo $(0.5 \mathrm{mg} / \mathrm{kg}$; Haldol, Janssen, Belgium) or saline (Sal, $0.9 \% \mathrm{NaCl}$ solution, Braun, Germany). The ip injection took place $60 \mathrm{~min}$ before the start of the playback experiment and during the time between the injection and the playback experiment, rats were kept singly (in a small cage with bedding and water ad libitum) in a dark room (according to Tonelli et al., 2017).

\section{0-kHz Ultrasonic Vocalizations Playback: Setup}

As experimental setups, an eight-arm radial maze (data sets 1 and 2) and a squared platform (data set 3), each elevated $52 \mathrm{~cm}$ above the ground, were employed. On two opposite sides of the given apparatus, an ultrasonic speaker (ScanSpeak, Avisoft Bioacoustics, Berlin, Germany) and an ultrasonic condenser microphone (CM16, Avisoft Bioacoustics) were placed $20 \mathrm{~cm}$ away from the end of the arm or platform. Only one of the speakers was active, whereas the other one served as a visual control. Experiments were conducted under red light ( $\sim 10$ lux).

\section{0-kHz Ultrasonic Vocalizations Playback: Acoustic Stimuli}

We presented two types of acoustic stimuli: (A) $50-\mathrm{kHz}$ USV recorded from an adult male WI rat (ca. $350 \mathrm{~g}$ ) during exploration of a cage containing scents from a recently removed cage mate (for details see Wöhr et al., 2008). This recording was composed of a sequence of $3.5 \mathrm{~s}$ with 13 different $50-\mathrm{kHz}$ calls (total calling time $0.9 \mathrm{~s}$ ) presented in a loop (for details see Wöhr and Schwarting, 2007). The peak amplitude was $70 \mathrm{~dB}$ (measured from a distance of $40 \mathrm{~cm}$ ), being in the typical range of 50-kHz USV (Kisko et al., 2020). (B) Time- and amplitudematched noise was generated with SASLab Pro (Version 4.2, Avisoft Bioacoustics) by replacing each $50-\mathrm{kHz}$ call by noise with matching duration and amplitude modulation. Accordingly, each noise playback series had the same temporal pattern and all call features were identical, except that the sound energy was not in a certain frequency range as in the natural $50-\mathrm{kHz}$ USV playback (for details see Wöhr and Schwarting, 2012). The acoustic stimuli were presented via an ultrasonic speaker (ScanSpeak, Avisoft Biosacoustics) with a frequency range of $1-120 \mathrm{kHz}$ and a flat frequency response $( \pm 12 \mathrm{~dB})$ between 15 and $80 \mathrm{kHz}$. Sounds were played via a portable ultrasonic power amplifier with a frequency range of $1-125 \mathrm{kHz}$ (Avisoft Bioacoustics) and via an external sound card with a sampling rate of $192 \mathrm{kHz}$ (Fire Wire Audio Capture FA-101, Edirol, London, United Kingdom).

\section{0-kHz Ultrasonic Vocalizations Playback: Paradigm}

At the beginning of the playback experiment, rats were placed individually in the center of the eight-arm radial maze (data sets 1 and 2) or the squared platform (data set 3). After an initial habituation period of $15 \mathrm{~min}$, the first playback presentation of 5 min duration commenced. The second playback presentation of 5 min duration followed after an inter-stimulus interval of $10 \mathrm{~min}$. Acoustic stimuli (i.e., $50-\mathrm{kHz}$ USV, noise) were presented in a counterbalanced manner. The trial ended with a poststimulus interval of $10 \mathrm{~min}$. The whole paradigm lasted $45 \mathrm{~min}$.

\section{Recording and Analysis of Response Calls}

For recording response calls emitted by the given experimental rat, two ultrasonic microphones were placed symmetrically on two sides of the maze (data sets 1 and 2) or the platform (data set 3) next to the speakers. They were connected via an UltraSoundGate $416 \mathrm{H}$ USB audio device (Avisoft Bioacoustics) to a computer, where acoustic data were recorded with a sampling rate of $250 \mathrm{kHz}$ (16-bit format; recording range $0-125 \mathrm{kHz}$ ) using RECORDER USGH (Avisoft Bioacoustics). For acoustical analysis, recordings were transferred to DeepSqueak (version 2.6.1, Windows standalone), a deep learning-based system for detection and analysis of USV (Coffey et al., 2019). Recorded files were converted into high-resolution spectrograms and were analyzed using the pre-trained automated "short rat call network 
V2." The settings for call detection were "high recall," with an overlap of $0.001 \mathrm{~s}$. This setting was chosen because it minimizes the possibility that a call is missed, albeit at the cost of false positives by including noise. Therefore, a custom trained network for denoising was applied afterward. The detected events were then transferred into the DeepSqueak Screener (Fork on GitHub by L. Lara-Valderrábano and R. Ciszek: 10.5281/zenodo.3690137), ${ }^{1}$ where the files were reviewed and denoised again manually by an experienced observer accepting (response calls) or rejecting (noise or playback calls) events. All response calls, irrespective of frequencies and durations, were counted. For later analysis, response calls during the 5 min before, during, and after the playback presentations (50-kHz USV or noise) were taken into account (referred to as stimulus phase). Outside this time window, calls occurred rarely. Acoustic features, i.e., call duration, peak frequency, and frequency modulation (difference between highest and lowest frequency), were defined and analyzed as described previously (Kisko et al., 2018). For classifying response calls, we applied previously established frequency thresholds (Brudzynski, 2001). Calls with frequencies higher than $32 \mathrm{kHz}$ were classified as $50-\mathrm{kHz}$ USV and calls below $32 \mathrm{kHz}$ were defined as $22-\mathrm{kHz}$ USV.

\section{Recording and Analysis of Overt Behavior}

As pointed out above, the behavioral data (locomotion, approach) were part of a recently published study focusing on the habituation of the social approach response to playback of 50$\mathrm{kHz}$ USV (Berz et al., 2021). Here, we reconsidered these data in the context of the new data on response calls in order to address the question whether locomotor activity and approach behavior evoked by playback of $50-\mathrm{kHz}$ USV are associated with the emission of response calls. Briefly, overt behavior was recorded and analyzed using EthoVision XT (Version 13, Noldus, The Netherlands). Locomotion was measured by the distance traveled. For quantifying approach behavior on the maze (data sets 1 and 2), the numbers of entries into the three arms proximal and distal to the active speaker and the time spent thereon were measured. For quantifying approach behavior on the platform (data set 3 ), it was virtually divided into 25 equal quadrants, with the six quadrants close to the active speaker serving as proximal zone, while the six quadrants close to the inactive speaker were defined as distal zones. Entries and time spent in these zones were measured (for details see Berz et al., 2021).

\section{Statistical Analysis}

Analyses of variance (ANOVAs) for repeated measurements were calculated with the between-subject factors playback order $(50-\mathrm{kHz}$ USV first vs. second), stocks (WI vs. SD), or drug treatment (Halo vs. Sal), and the within-subject factors stimulus phase ( 5 min before, during, or after playback) and playback stimulus $(50-\mathrm{kHz}$ USV or time- and amplitudematched noise). This was followed by two-tailed $t$-tests for comparing individual experimental groups. The ratio between calling and non-calling rats was evaluated by a $\chi^{2}$-test (calculated

${ }^{1}$ https://github.com/UEFepilepsyAIVI/DeepSqueak.git using https://www.socscistatistics.com/tests/chisquare2/default2. aspx). Approach behavior was quantified by subtracting the times spent on proximal arms (or in proximal zones) before the $5 \mathrm{~min}$ of $50-\mathrm{kHz}$ USV playback from the time spent there during the 5 min of playback. The same was done with the entries into proximal arms or zones. Pearson correlation coefficients (bivariate) were calculated for the correlation between numbers of emitted calls and approach behavior. For testing a possible correlation with locomotor behavior, locomotion (distance traveled in $\mathrm{cm}$ ) during the $5 \mathrm{~min}$ before playback were subtracted from that during the $5 \mathrm{~min}$ during playback. This number was then correlated with the numbers of response calls emitted using the Pearson correlation coefficient. For general locomotor activity correlations, the distance traveled during the initial 15min habituation period were taken into account. All $t$-tests, ANOVAs, and correlations were calculated with IBM SPSS Statistics (version 25). Graphs were made using GraphPad Prism (version 8). Data are represented as means \pm SEM (standard error of mean). A $p$-value of $<0.050$ was considered statistically significant.

\section{RESULTS}

\section{Data Set 1: Response Calls Call Numbers and Latencies}

Playback of $50-\mathrm{kHz}$ USV induced response calls in the majority of WI rats. Among the 24 rats of data set 1, 23 of them emitted response calls. The mean number of response calls was $123.5 \pm 26.21$, ranging between 0 and 414 calls in total per rat (Figure 1A). During the $5 \mathrm{~min}$ before $50 \mathrm{kHz}$ USV playback, no calls were emitted. The occurrence of response calls was not dependent on whether $50-\mathrm{kHz}$ USV were presented as the first or the second stimulus $\left(t_{22}=0.82, p=0.21\right)$. Importantly, high levels of response calls were emitted specifically in reaction toward playback of $50-\mathrm{kHz}$ USV but not noise, irrespective of whether $50-\mathrm{kHz}$ USV were presented as the first $\left(t_{11}=2.8, p=0.017\right)$ or the second stimulus ( $t_{11}=4.013, p=0.002$; Figure 1A). The latency to start calling after onset of $50-\mathrm{kHz}$ USV was $20.17 \pm 88.17 \mathrm{~s}$ (Figure 1B). Stimulus order did not affect call latency $\left(t_{21}=0.52\right.$, $p=0.61$ ). Therefore, we abstained from differentially considering stimulus order further in all following analyses.

\section{Data Set 2: Stock Differences Call Numbers and Latencies}

Consistent with data set 1 , response calls were seen in the majority of rats in data set 2 focusing on possible stock differences between $\mathrm{WI}$ and SD rats. From the two different stocks, 10 out of the $18 \mathrm{WI}$ rats emitted calls in response to $50-\mathrm{kHz}$ USV playback and 12 out of $18 \mathrm{SD}$ rats did. The ratios between calling and non-calling rats did not differ between stocks $\left(x^{2}{ }_{1,36}=0.468, p=0.49\right)$. Likewise, the mean numbers of response calls (Figure $2 \mathrm{~A} ; t_{34}=0.032$, $p=0.975$; WI: $44.39 \pm 17.81$; SD: $45.17 \pm 16.45)$ as well as the latencies to start calling (Figure 2B; $t_{20}=0.547, p=0.590$; WI: $50.56 \pm 41.16 \mathrm{~s}$; SD: $29.88 \pm 4.93 \mathrm{~s}$ ) did not differ between WI and SD. In both stocks, high levels of response calls were exclusively evoked by playback of $50-\mathrm{kHz}$ USV, while response calls rarely 

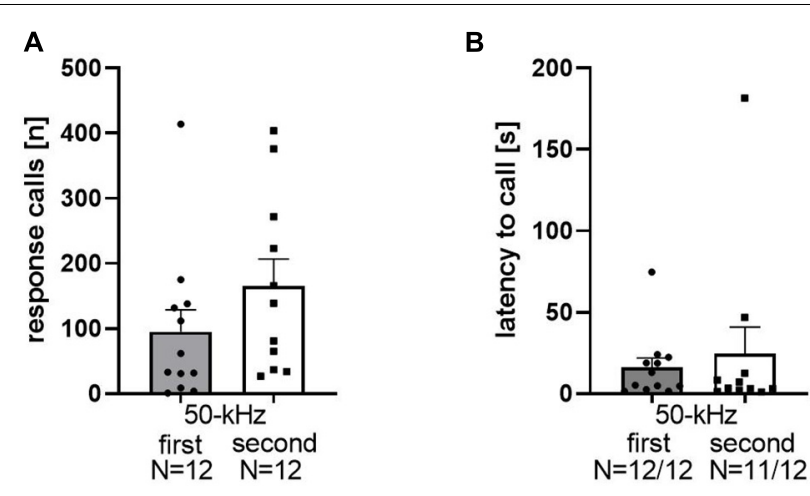

FIGURE 1 | Total numbers of response calls emitted (A) for playback of $50-\mathrm{kHz}$ as the first stimulus [95.25 \pm 33.62 (mean \pm SEM)] or as the second stimulus (152 \pm 40.07 ) of WI rats. Latencies after stimulus onset (B): $50-\mathrm{kHz}$ first: $16.04 \pm 5.67 ; 50-\mathrm{kHz}$ second: $24.67 \pm 16.19$.

occurred during noise playback (WI: $t_{17}=2.717, p=0.015$; SD: $\left.t_{17}=2.727, p=0.014\right)$.

\section{Data Set 1 and 2: Detailed Analyses Temporal Emission Pattern}

We next pooled the data sets 1 and 2 and performed more detailed analyses. First, a detailed temporal analysis revealed that the emission of response calls was strongly dependent on stimulus $\left(F_{1}, 58=21.260, p<0.001\right)$ and stimulus phase $\left(F_{2}, 116=21.120, p<0.001\right)$, with an interaction between stimulus and stimulus phase $\left(F_{2}, 116=21.002, p<0.001\right)$, while stock had no major impact (stock: $F_{1}, 58=2.311, p=0.134$; stock $\times$ stimulus: $F_{1}, 58=2.253, p=0.139$; stock $\times$ stimulus phase: $F_{2}, 116=2.308, p=0.104$; stock $\times$ stimulus $\times$ stimulus phase: $F_{1}, 116=2.290, p=0.106$; Figure 3). Specifically, playback of $50-\mathrm{kHz}$ USV but not noise led to a prominent increase in response calls, which occurred during the $5 \mathrm{~min}$ of $50-\mathrm{kHz}$ USV playback and up to $5 \mathrm{~min}$ thereafter. The peak of vocalization typically occurred in the second or third minute after $50-\mathrm{kHz}$ USV playback onset. With onset of the $50-\mathrm{kHz}$ USV playback, the numbers of emitted response calls increased significantly in WI $\left(F_{1}, 41=27.940, p<0.001\right)$ and $\operatorname{SD}$ rats $\left(F_{1}, 17=7.436\right.$, $p=0.014)$. After that, calling rate decreased to zero at the latest 5 min after the playback had ended. In both stocks, substantial calling only occurred in response to $50-\mathrm{kHz}$ USV playback and not in response to noise, reflecting high specificity of response call emission (WI: $F_{1}, 41=25.387, p<0.001$; SD: $F_{1}, 17=7.538$, $p=0.014)$. Furthermore, the call emission sequence showed that most animals started calling with higher frequencies around $50 \mathrm{kHz}$ and quickly changed to emit calls of frequencies around $22 \mathrm{kHz}$ (Supplementary Figure 1A).

\section{Response Call Features}

Secondly, detailed analyses of acoustic features revealed that the calls in response to $50-\mathrm{kHz}$ USV playback were heterogeneous since they were characterized by a large variability in acoustic features and shapes. Both, WI and SD rats emitted calls below and above $32 \mathrm{kHz}$. These calls had rather different

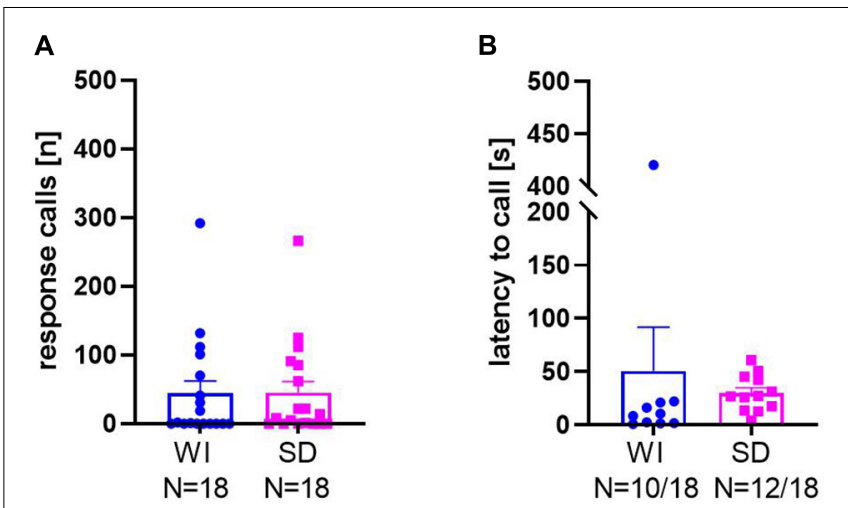

FIGURE 2 | Total numbers of response calls (A) and latencies to call (B) in Wistar (WI) and Sprague-Dawley (SD) rats.

durations and shapes, and the temporal spaces between them varied substantially.

For a further quantification of the response calls, mean peak frequencies, mean call durations, and mean frequency modulations were quantified (Figure 4; for examples of response calls, see Figure 5). Peak frequencies of WI rats $(32.48 \pm 1.46 \mathrm{kHz})$ and SD rats $(37.82 \pm 3.2 \mathrm{kHz}$; Figure $4 \mathrm{~A}) \mathrm{did}$ not differ significantly from each other $\left(t_{15 \cdot 82}=1.52, p=0.149\right)$. Call durations of WI rats $(0.34 \pm 0.03 \mathrm{~s})$ tended to be longer than those of SD rats $\left(0.24 \pm 0.05 s ; t_{43}=1.859, p=0.07\right)$. Frequency modulations did not differ between stocks $\left(t_{43}=0.98, p=0.33\right.$; WI: $6.68 \pm 0.51 \mathrm{kHz}$; SD: $7.68 \pm 0.97 \mathrm{kHz}$ ).

To visualize the different call parameters and the distribution of individual calls, scatter plots for either call durations or frequency modulations were plotted vs. peak frequencies (Figure 6). This analysis showed that most calls were below $32 \mathrm{kHz}$, with durations above and below $0.3 \mathrm{~s}$. Frequency modulations were mainly below $5 \mathrm{kHz}$. The main distribution of the calls was around mean peak frequencies below $32 \mathrm{kHz}$ in both stocks, but in SD rats also another distribution peak occurred around $50 \mathrm{kHz}$, with call durations typically shorter than $0.3 \mathrm{~s}$ and frequency modulations below $5 \mathrm{kHz}$ (Figures 6B,D).

Next, we quantified call numbers depending on acoustic call features and divided response calls into those with mean peak frequencies below or above $32 \mathrm{kHz}$, durations shorter or longer than $0.3 \mathrm{~s}$, and frequency modulations below or above $5 \mathrm{kHz}$ (Table 1). This analysis showed that in both stocks the majority of response calls was below $32 \mathrm{kHz}$. Considering durations, most calls were shorter than $0.3 \mathrm{~s}$, particularly in SD rats. Frequency modulations were mainly below $5 \mathrm{kHz}$. When comparing the percentages of calls with mean peak frequencies below $32 \mathrm{kHz}$ among stocks, WI rats were found to have higher percentages of calls below $32 \mathrm{kHz}\left(t_{43}=2.137, p=0.038\right)$. Considering percentages of calls with durations below $0.3 \mathrm{~s}$, stocks did not differ $\left(t_{43}=-1.95, p=0.058\right)$. The same was true for frequency modulations. Similar percentages of calls were emitted with modulations below $5 \mathrm{kHz}$ in both stocks $\left(t_{43}=0.173, p=0.864\right)$.

In addition, we asked whether response calls below or above $32 \mathrm{kHz}$ were related to each other in individual 


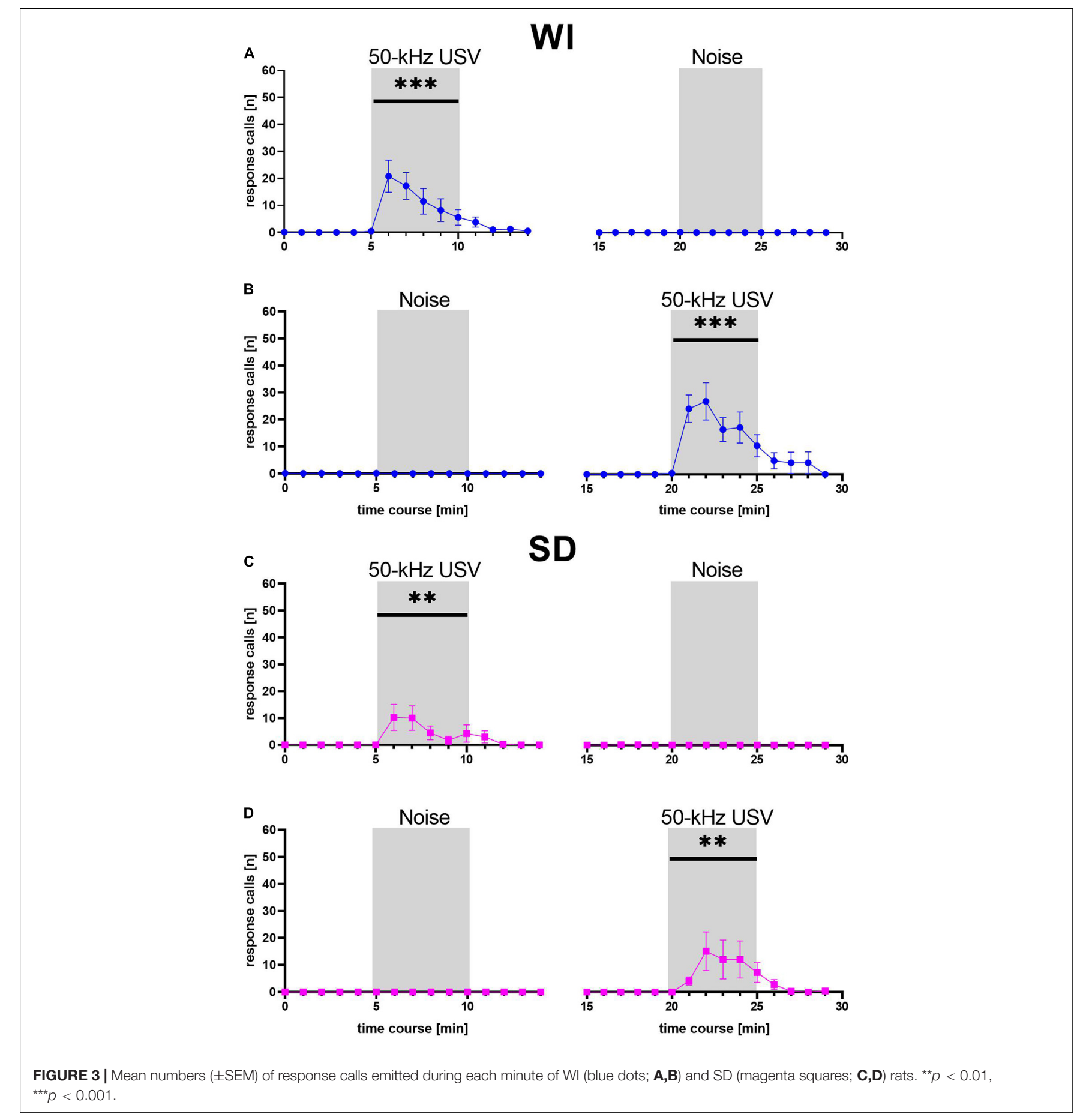

animals (Figure 7), but did not find significant correlations between the two in WI $(r=0.08, p=0.66)$ or $\mathrm{SD}$ rats $(r=-0.26, p=0.44)$.

\section{Relationships Between Response Calls and Playback-Induced Approach}

Thirdly, we asked whether the emission of the response calls was correlated with social approach behavior evoked by playback of $50-\mathrm{kHz}$ USV. As stated in the Introduction, the present response call data sets were obtained in a study where social approach behavior evoked by $50-\mathrm{kHz}$ USV playback was examined (Berz et al., 2021). In that study, approach behavior was quantified by subtracting the time spent on the proximal arms (i.e., close to the speaker) before playback from the time spent thereon during the presentation of $50-\mathrm{kHz}$ USV. The same was done for the proximal entries (see detailed analysis in Berz et al., 2021). These numbers were now correlated with the total amount of response calls evoked by playback of $50-\mathrm{kHz}$ USV to see 

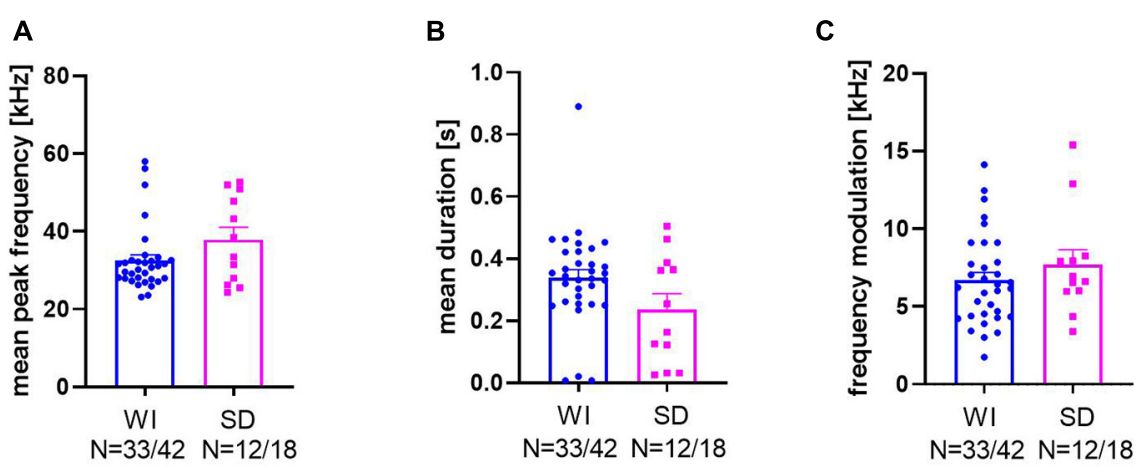

FIGURE 4 | Bar graphs and individual data points of mean peak frequency (A), mean duration (B), and frequency modulation (C) of WI (blue dots) and SD rats (magenta squares).

whether social approach behavior was related to the emission of response calls across individual rats. In WI rats, this tended to be the case. The more time the rats spent close to the active speaker, the more calls in response to $50-\mathrm{kHz}$ USV playback they tended to emit $(r=0.314, p=0.075)$. A more prominent correlation was evident in SD rats, where social approach and the emission of response calls were strongly associated (SD: $r=0.662, p=0.019)$. No such correlations were found with respect to proximal arm entries (WI: $r=0.01, p=0.952$; SD: $r=-0.017, p=0.948)$. To test whether these correlations were only a byproduct of locomotor activity during playback, the total numbers of response calls were correlated with the degree of locomotor activation using the distance traveled during playback in comparison to the distance traveled before playback. Neither in WI nor SD rats a correlation was found $(r=0.065$, $p=0.681 ; r=0.151, p=0.551$, respectively). Also, the numbers of response calls were not correlated with locomotor activity during the first $15 \mathrm{~min}$ on the maze as a measure of general locomotor activity (WI: $r=0.031, p=0.864$; SD: $r=0.187$, $p=0.540)$.

\section{Data Set 3: Effects of Drug Treatment Call Numbers and Latencies}

In the third data set, rats were treated either with the dopaminergic D2 receptor antagonist Halo or saline as a control. The pharmacological treatment had no prominent effect on the emission of response calls and the proportion of vocalizing rats (saline: 15 out of 24, Halo: 20 out of 24) did not differ between Sal and Halo $\left(x^{2}{ }_{1}, 48=2.64, p=0.104\right)$. Moreover, treatment did not affect response call numbers $\left(t_{46}=0.465, p=0.644\right.$; Figure 8A; Sal: $66.5 \pm 31.18$; Halo: $86 \pm 31.53)$ and latencies to start calling $\left(t_{33}=0.578, p=0.567\right.$; Figure 8B; Sal: $19.41 \pm 4.18$ s; Halo: $26.33 \pm 9.86 \mathrm{~s})$.

\section{Temporal Emission Pattern}

Similar to the previous data sets 1 and 2, the emission of response calls was strongly dependent on stimulus $\left(F_{1}, 46=11.771\right.$, $p=0.001)$ and stimulus phase $\left(F_{2}, 92=14.443, p<0.001\right)$, with an interaction between stimulus and stimulus phase $\left(F_{2}\right.$, $92=14.373, p<0.001)$, while treatment had no major impact (treatment: $F_{1},{ }_{46}=0.194, p=0.662$; treatment $\times$ stimulus: $F_{1}$, ${ }_{46}=0.232, p=0.632$; treatment $\times$ stimulus phase: $F_{2}, 92=0.842$, $p=0.434$; treatment $\times$ stimulus $\times$ stimulus phase: $F_{1}, 92=0.797$, $p=0.454$; Figure 9). Specifically, as in the previous data sets 1 and 2, playback of $50-\mathrm{kHz}$ USV but not noise led to a prominent increase in response calls, which occurred during the $5 \mathrm{~min}$ of $50-\mathrm{kHz}$ USV playback and up to $5 \mathrm{~min}$ thereafter. The peak was again typically seen during the second or third minute after 50$\mathrm{kHz}$ USV playback onset. With onset of $50-\mathrm{kHz}$ USV playback, the numbers of emitted response calls increased significantly in rats treated with $\operatorname{Sal}\left(F_{1}, 23=6.443, p=0.018\right)$ but also in rats treated with Halo $\left(F_{1}, 23=8.068, p=0.009\right)$. After that, calling rate decreased to zero at the latest $5 \mathrm{~min}$ after playback had ended. Substantial calling only occurred in response to $50-\mathrm{kHz}$ USV and not in response to noise and was therefore specific to the 50$\mathrm{kHz}$ USV playback in both treatment groups (Sal: $F_{1}, 23=4.687$, $p=0.041$; Halo: $F_{1}, 18=7.613, p=0.013$ ). Furthermore, the call emission sequence showed that most animals started calling with higher frequencies around $50 \mathrm{kHz}$ and quickly changed to emit calls of frequencies around $22 \mathrm{kHz}$ (Supplementary Figure 1B).

\section{Response Call Features}

For a further characterization of response calls in the third data set, their mean peak frequencies, durations, and frequency modulations were analyzed. Sal-treated animals had peak frequencies around $33.76 \pm 2.8 \mathrm{kHz}$, which was not significantly different from Halo-treated animals (30.89 $\pm 2.49 \mathrm{kHz}$; $t_{33}=0.898, p=0.376$; Figure 10A). Call durations in controls were $0.282 \pm 0.036 \mathrm{~s}$, which was significantly shorter than those of Halo-treated rats $\left(0.395 \pm 0.039 \mathrm{~s} ; t_{33}=2.048, p=0.049\right.$, Figure 10B). Frequency modulation did not differ between treatment groups and Sal-treated rats called with a frequency modulation of $5.33 \pm 0.56 \mathrm{kHz}$ compared to $6.16 \pm 0.66 \mathrm{kHz}$ in HALO-treated rats $\left(t_{33}=0.919, p=0.365\right.$; Figure 10C).

The response calls were various in shape and differed in call parameters (for examples of response calls, see Figure 11). For better visualization of the different call parameters and the distribution of the individual calls, scatter plots for either call durations or frequency modulations were plotted vs. peak frequencies (Figure 12). The accompanying histograms show 


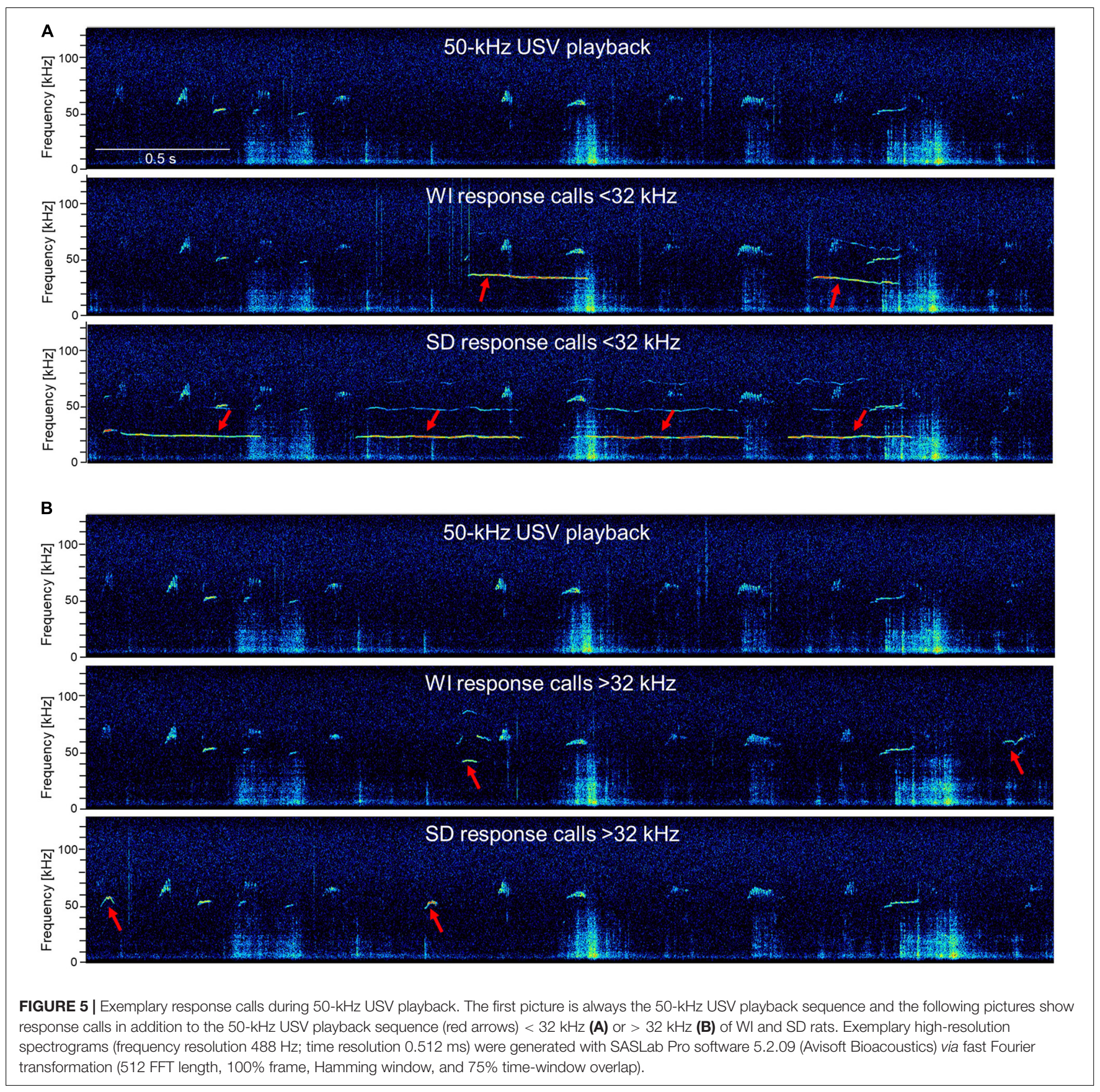

the main distribution at mean peak frequencies around $25 \mathrm{kHz}$ in both treatment groups; meaning that the majority of calls were below $32 \mathrm{kHz}$. Especially in Halo-treated rats, few calls were above $32 \mathrm{kHz}$. Call durations were as well above as below $0.3 \mathrm{~s}$ in Sal- and Halo-treated rats. Frequency modulation was mainly below $5 \mathrm{kHz}$.

Next, we again quantified call numbers depending on acoustic call features and divided response calls into those with mean peak frequencies below or above $32 \mathrm{kHz}$, durations shorter or longer than $0.3 \mathrm{~s}$, and frequency modulations below or above $5 \mathrm{kHz}$ (Table 2). When comparing the percentages of calls with mean peak frequencies below $32 \mathrm{kHz}$ among treatment groups, no significant difference was detected $\left(t_{33}=-0.978\right.$, $p=0.335$ ). Considering durations below $0.3 \mathrm{~s}$, there was likewise no difference $\left(t_{33}=1.996, p=0.054\right)$. The same was true for frequency modulations, since similar percentages of calls were emitted with modulations smaller than $5 \mathrm{kHz}$ in both groups $\left(t_{33}=0.979, p=0.335\right)$.

In addition, we again asked whether response calls below or above $32 \mathrm{kHz}$ were related in individual animals (Figure 13), but found no significant correlations in Sal- $(r=-0.161, p=0.566)$ or Halo-treated rats $(r=0.123, p=0.606)$. 


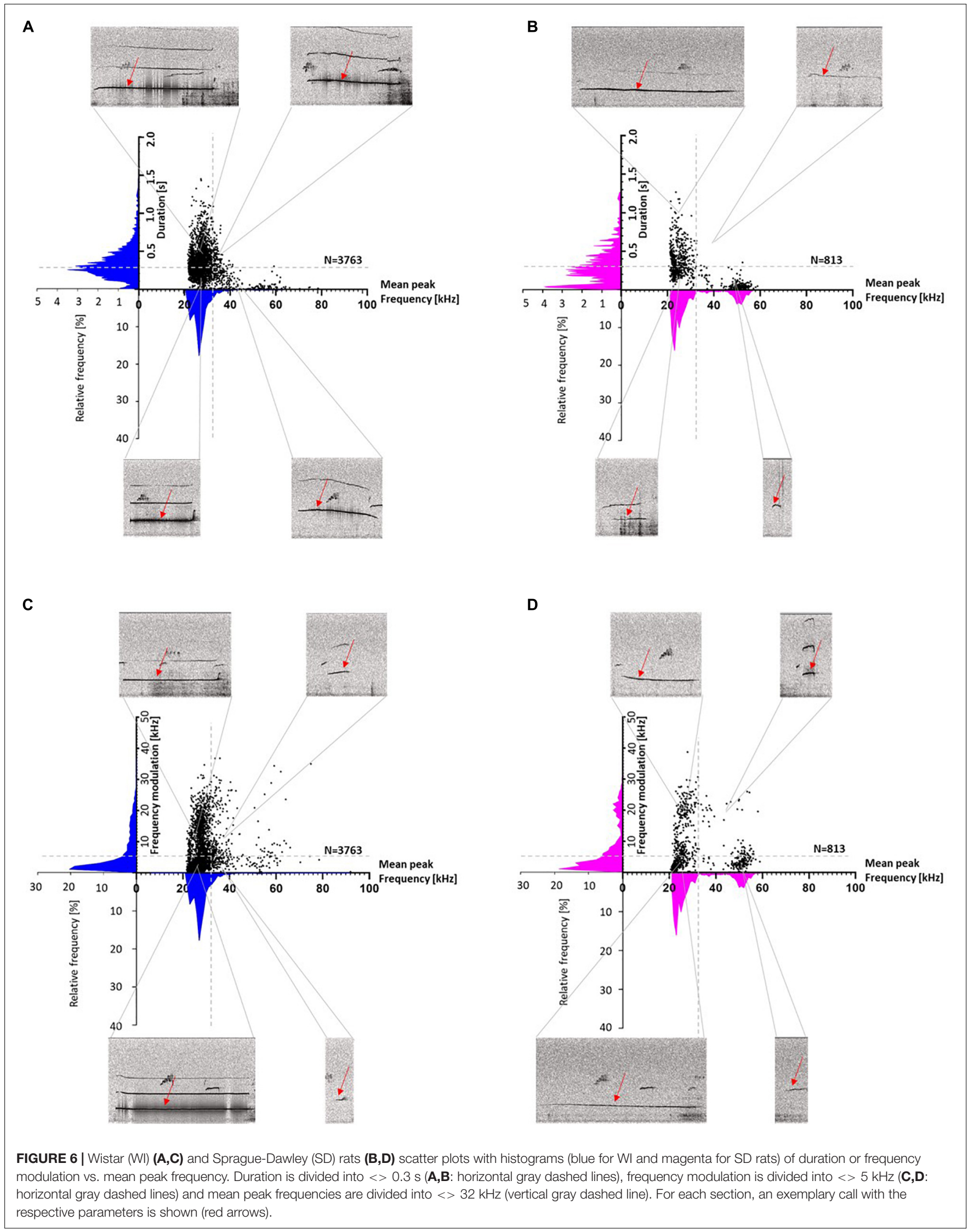


TABLE 1 | Scatter plot distributions for Wistar (WI) and Sprague-Dawley (SD) rats.

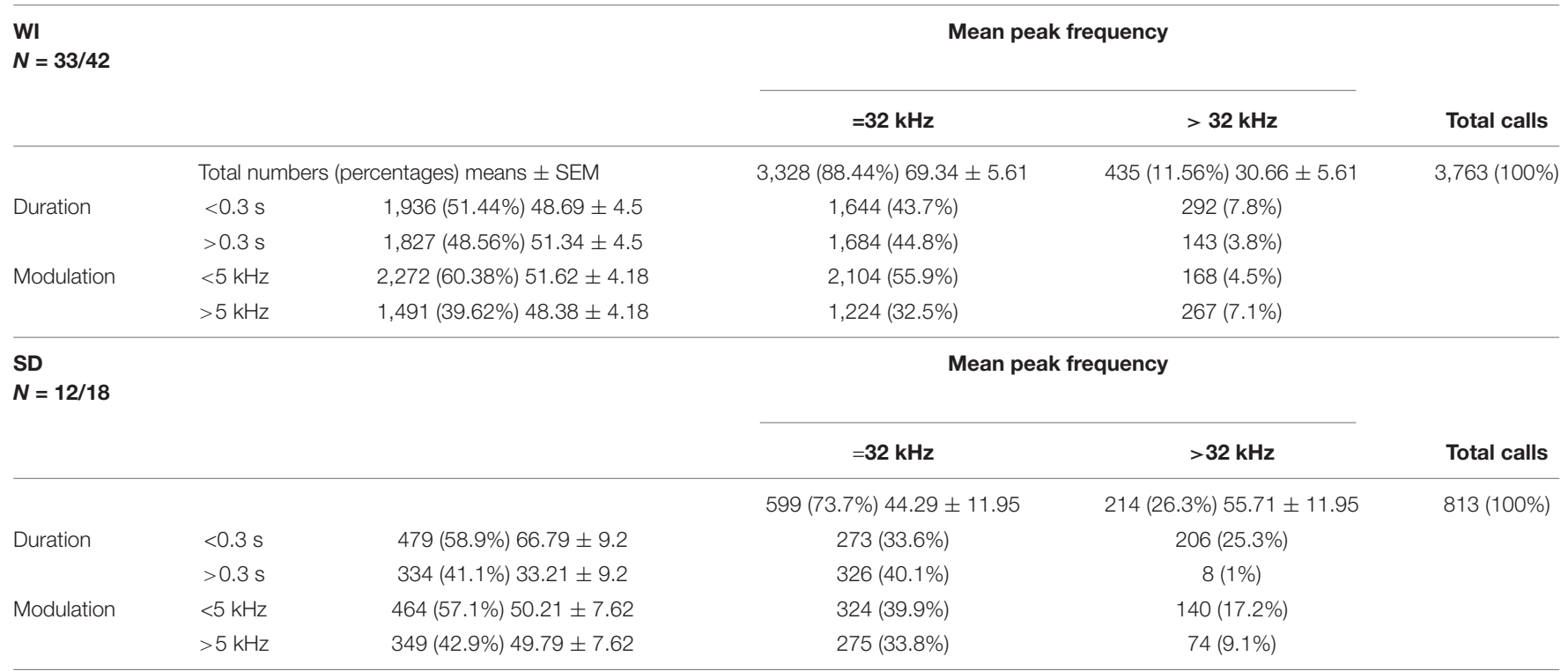

Mean peak frequencies $<$ or $>32 \mathrm{kHz}$, Durations $=$ or $>0.3 \mathrm{~s}$, frequency modulations $=$ or $>5 \mathrm{kHz}$.
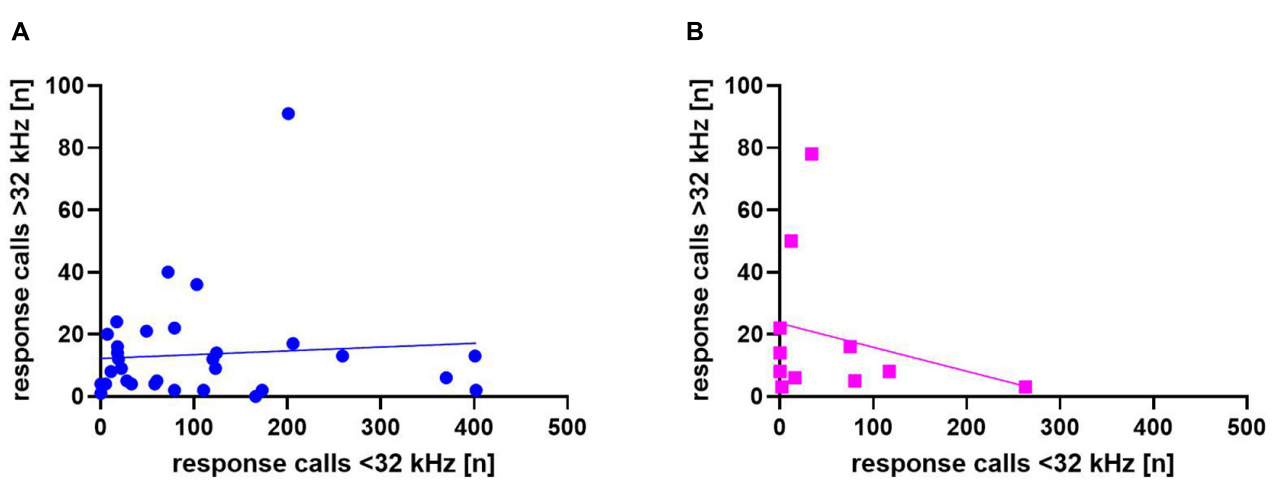

FIGURE 7 | Correlation between calls $<32 \mathrm{kHz}$ and $>32 \mathrm{kHz}$ for Wistar (WI) (A) and Sprague-Dawley (SD) (B) rats. Each data point represents response calls below and above $32 \mathrm{kHz}$ of one animal.

\section{Relationships Between Response Calls and Playback-Induced Approach}

To see whether social approach was associated with the emission of response calls, these two parameters were again correlated. The results were the same in both treatment conditions. In Sal-treated rats, there were no significant correlations, neither between the time spent in the proximal arms close to the active speaker nor between the entries into those with the amount of response calls (Sal time: $r=-0.0195, p=0.487$; Sal entries: $r=0.059, p=0.783)$. In Halo-treated animals, likewise no significant correlations between proximal time or entries and number of emitted calls were detected (Halo time: $r=0.143, p=0.547$; Halo entries: $r=-0.112, p=0.602$ ). Moreover, locomotor activity during $50 \mathrm{-kHz}$ USV playback in comparison to the distance traveled before playback was not correlated with the total numbers of response calls, irrespective of treatment condition (Sal: $r=-0.101, p=0.639$; Halo: $r=-0.113, p=0.598)$. In addition, locomotor activity during the first $15 \mathrm{~min}$ on the platform was not correlated with the number of response calls (Sal: $r=-0.224, p=0.421$; Halo: $r=0.238, p=0.312$ ).

\section{DISCUSSION}

In this study, we characterized response calls emitted by rats exposed to playback of appetitive $50-\mathrm{kHz}$ USV, previously shown to function as social contact calls (Wöhr, 2018). The phenomenon that rats respond to playback of species-specific 50$\mathrm{kHz}$ calls by emitting response calls has been repeatedly reported before, but has not been described in detail yet (Wöhr and Schwarting, 2007, 2009; Willadsen et al., 2014; Willuhn et al., 2014; Engelhardt et al., 2017, 2018; Berg et al., 2018, 2021; Kisko et al., 2020; Olszyński et al., 2020, 2021; for an overview see Supplementary Table 1). First, we described the emission of response calls in reaction toward $50-\mathrm{kHz}$ USV playback in 

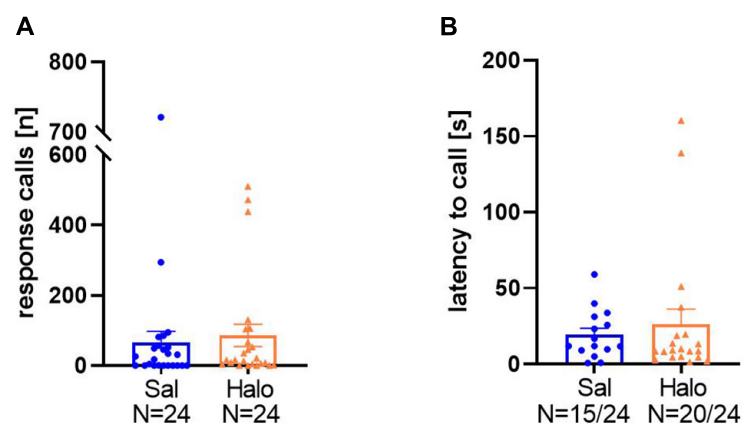

FIGURE 8 | Total numbers of response calls (A) and latencies to call (B) in Sal- and Halo-treated rats. Data are presented as individual results and as means \pm SEM.

WI rats. Secondly, we compared these to SD rats. Thirdly, we analyzed the effect of blocking DA receptors on response calls using Halo, as compared to vehicle-injected WI subjects.

Through these means, we could demonstrate that most rats emitted response calls. Importantly, the emission of response calls was clearly linked to $50-\mathrm{kHz}$ USV playback. In fact, response calls were seen specifically in response to $50-\mathrm{kHz}$ USV but not in response to time- and amplitude-matched noise, replicating previous results (Willadsen et al., 2014; Willuhn et al., 2014; Engelhardt et al., 2017, 2018; Berg et al., 2018, 2021; Kisko et al., 2020; Olszyński et al., 2020, 2021). When exposed to 50-kHz USV, receiver rats often started emitting response calls within the first minute of playback and emission rates were typically peaking after around 2-3 min, often outlasting playback for up to $5 \mathrm{~min}$. This certainly supports naming these calls "response calls."

Most response calls were characterized by peak frequencies below $32 \mathrm{kHz}$, the threshold typically applied to differentiate between 22- and 50-kHz USV (Brudzynski, 2001). Although peak frequencies were highly variable and ranged roughly between 20 and $80 \mathrm{kHz}$, the vast majority of response calls occurred in a frequency range of 20-32 kHz. Similarly, call durations were characterized by large variability, ranging from a few milliseconds to up to $1.5 \mathrm{~s}$. Call durations of about $0.3 \mathrm{~s}$ occurred at a particularly high rate. Frequency modulations were typically below $5 \mathrm{kHz}$. When comparing these values to the parameters of typical 22- and $50-\mathrm{kHz}$ USV, our values correspond more to 22$\mathrm{kHz}$ USV; more precisely the short $22-\mathrm{kHz}$ USV type since the durations were rarely longer than $0.3 \mathrm{~s}$ (Brudzynski, 2021).

The emission of response calls was seen in WI and SD rats, suggesting that this is a robust phenomenon not dependent on stocks. Specifically, we found that there were no substantial differences between WI and SD rats, concerning numbers of emitted calls, latencies to start calling, and call likelihood. In both stocks there was a large variability among response calls. However, their mean peak frequencies, call durations, and frequency modulations did not differ significantly between experimental conditions. SD rats only differed in one aspect by clearly showing calls around frequencies of $50 \mathrm{kHz}$, which was not that prominent in WI rats. This is somehow in line with other studies that also showed higher emission of $50-\mathrm{kHz}$ USV and elevated rough-and-tumble play behavior in SD compared to WI rats (Manduca et al., 2014). Other studies, however, found that WI rats emitted more $50-\mathrm{kHz}$ USV compared to SD rats (Schwarting, 2018a,b), indicating that WI rats are more prone to emit USV in general, which is also not represented by our data. If at all, on a descriptive level, WI rats emit slightly less response calls compared to SD rats. Regarding call parameters, previous studies showed marginal differences between stocks, i.e., shorter call durations in SD rats compared to WI rats (Schwarting, 2018b). On a descriptive level again, this aligns with our results, albeit this difference in call duration did not yield significance. Apart from stock differences, various other factors like breeding or experience have to be taken into account. Moreover, interindividual differences should not be neglected, as our results also suggest (Schwarting, 2018a,b).

In our study, the pharmacological treatment with the D2 antagonist Halo did not affect call likelihood, call rates, latencies, temporal distribution, peak frequency, and frequency modulation. In Sal-treated WI rats, the majority of calls was again below $32 \mathrm{kHz}$, however, in Halo-treated rats this was even more prominent and Halo treatment also led to longer call durations. Previous studies showed that exposure to 50$\mathrm{kHz}$ USV playback under the influence of systemically applied amphetamine, a catecholaminergic agonist, resulted in response calls with frequencies around $50 \mathrm{kHz}$ at the expense of $22 \mathrm{kHz}$ (Engelhardt et al., 2017). Specifically, calls of lower frequencies decreased drastically under the influence of amphetamine. In contrast, response calls in the $50 \mathrm{kHz}$ range increased dosedependently following amphetamine administration. This is in line with a large number of studies showing that the emission of 22 - and $50-\mathrm{kHz}$ USV are associated with the activation of distinct neurotransmitter systems (for review: Brudzynski, 2021). While $22-\mathrm{kHz}$ USV are associated with the cholinergic system (Brudzynski, 2001; Kroes et al., 2007; Willadsen et al., 2018), the dopaminergic system plays an important role in the regulation of $50-\mathrm{kHz}$ USV (Wöhr, 2021). For instance, electrolytic or 6hydroxydopamine lesions of the ventral tegmental area reduce the emission of 50-kHz USV (Burgdorf et al., 2007). Conversely, emission of $50-\mathrm{kHz}$ USV can be evoked by electrical stimulation of the ventral tegmental area or the nucleus accumbens (Burgdorf et al., 2000, 2007). Moreover, psychostimulants, most notably amphetamine, lead to a robust increase in $50-\mathrm{kHz}$ USV emission (Rippberger et al., 2015). Additionally, playback of 50-kHz USV was shown to induce enhanced levels of activity in the nucleus accumbens (Sadananda et al., 2008), where it elicits a rapid phasic release of dopamine (Willuhn et al., 2014). Based on these findings, one could have assumed that the dopaminergic receptor blockade with Halo should decrease response call numbers, especially those above $32 \mathrm{kHz}$, which was apparently not the case. Possibly, these calls are not critically dependent on dopamine D2 receptor function, and might be dependent on endogenous opiates, as indicated by an earlier playback study with the opiate receptor antagonist naloxone (Wöhr and Schwarting, 2009).

Together, the present findings indicate that the emission of response calls is a robust phenomenon that is seen specifically in response to playback of $50-\mathrm{kHz}$ USV independent of stock and despite blocking dopamine neurotransmission. These 

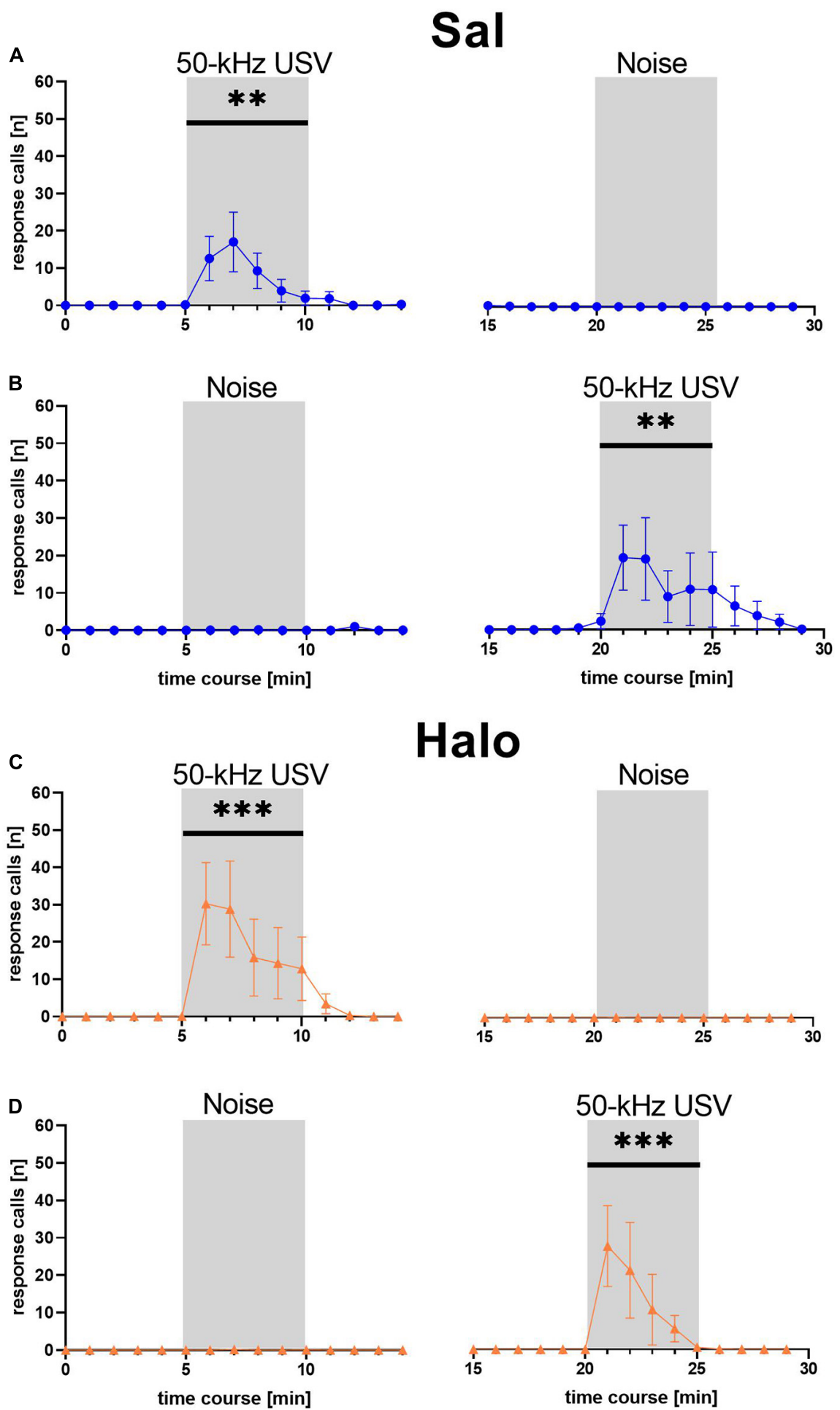

FIGURE 9 | Mean number of response calls emitted during each minute of Sal- (blue; A,B) and Halo-treated (orange; C,D) rats. Most calls were emitted during $50-\mathrm{kHz}$ USV stimulus and almost no calls were emitted during noise. ${ }^{* \star} p<0.01,{ }^{\star \star \star} p<0.001$.

observations are in line with the idea that the emission of response calls reflects changes in affect that are caused by playback of $50-\mathrm{kHz}$ USV. For example, one might expect the induction of a positive affective state in response to appetitive $50-\mathrm{kHz}$ USV. On the other hand, it was suggested that response calls reflect frustration induced by the inability to reach the 

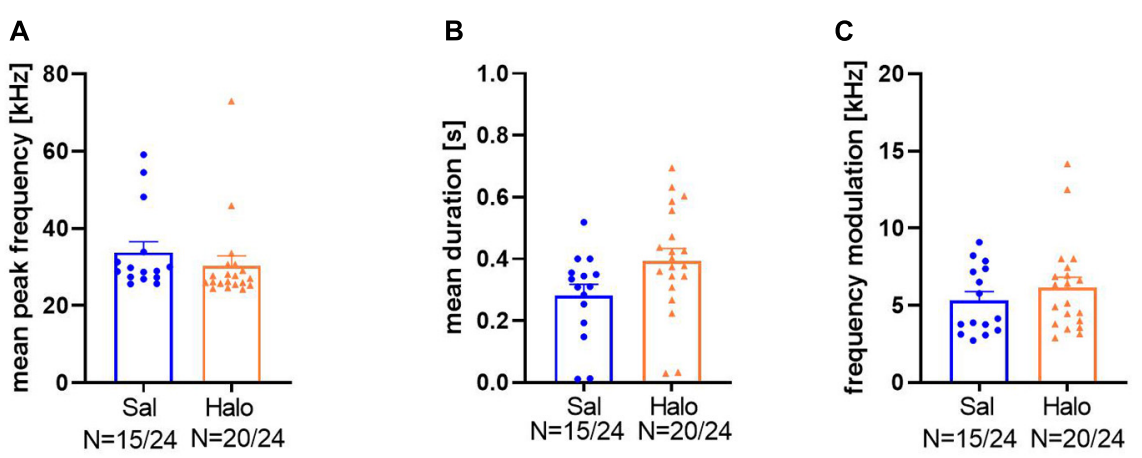

FIGURE $\mathbf{1 0}$ | Call parameters of Sal- (blue) and Halo-treated (orange) rats for mean peak frequency (A), mean duration (B), and frequency modulation (C)

conspecific emitting $50-\mathrm{kHz}$ USV. Alternatively, response calls might serve communicative functions as social contact calls or as appeasement signals. While the present findings do not allow drawing strong conclusions about causes and functional significance of response calls, they provide first insights into potential mechanisms underlying their emission.

In support of the idea that response calls might reflect an affective state we hypothesize that the rats are not solely in one affective state, but rather in an ambivalent state. There is convincing evidence in support of the notion that USV emission reflects prominent affective states (Brudzynski, 2021) and that different call types are associated with distinct states (Brudzynski, 2013b). Because USV below $32 \mathrm{kHz}$ are typically believed to function as alarm or distress calls reflecting a negative affective state, this would suggest that playback of $50-\mathrm{kHz}$ USV induced a negative state in the receiver rats. However, the strong level of social approach behavior and the emission of $50-\mathrm{kHz}$ response calls, at least in SD rats, evoked by playback of $50-\mathrm{kHz}$ USV speaks against the induction of a solely negative affective state through 50-kHz USV playback (Wöhr, 2018). Furthermore, the positive and negative emotional states in rats were proposed to be mutually exclusive and acting in an antagonistic manner (Brudzynski, 2021). It is possible, however, that the two states quickly alternate which leads to the hypothesis of an ambivalent state, with negative and positive phases present in an oscillating manner. This is also reminiscent of an approach/avoidance conflict, i.e., a situation characterized by choices leading to either reward or punishment (Aupperle et al., 2015). Interestingly, it was shown that rats emit $22-\mathrm{kHz}$ as well as $50-\mathrm{kHz}$ USV during neutral situations and not only aversive ones (Robakiewicz et al., 2019). The study by Robakiewicz et al. (2019) also showed that both call types and hence presumably both emotional states can be present during an emotional neutral task of performing nose pokes in order to change the light of the experimental apparatus. Both call types were also found in a cocaine self-administration task (Barker et al., 2010), where animals received either high or low doses of cocaine. Low dose rats predominantly emitted short $22-\mathrm{kHz}$ calls and high dose rats emitted mostly $50-\mathrm{kHz}$ calls. Nevertheless, both groups showed calls of both emotional states and this supports the hypothesis of the ambivalent state. In the present study, however, only SD rats emitted $50-\mathrm{kHz}$ USV to a higher extent and all other experimental groups mainly emitted calls with frequencies below $32 \mathrm{kHz}$. Additionally, the emissions of response calls below and above $32 \mathrm{kHz}$ were not correlated across individual rats, suggesting that there was no general tendency for emitting response calls in both frequency ranges, which speaks against the hypothesis of an ambivalent state.

With respect to the emission of $22-\mathrm{kHz}$ calls, this phenomenon might be explained by the hypothesis of a frustrated state in the receiver rat, possibly induced by the violated expectation of another rat being present. Other studies suggested that short $22-\mathrm{kHz}$ calls $(<0.3 \mathrm{~s})$ represent a dysphoric state or displeasure without any external threat (Simmons et al., 2018), which is in line with the mean peak frequencies, durations, and low frequency modulations of the response calls found in our study. This might also be an indication that calls with low frequencies in response toward $50-\mathrm{kHz}$ USV playback are an expression of internal distress, i.e., frustration, as suggested before (Wöhr and Schwarting, 2009). Frustration is defined as a result of behavior after an expected but not received reward (Scull et al., 1970; Burokas et al., 2012). In our playback paradigm, the rat probably realized that there was no rat physically present for interaction after hearing the $50-\mathrm{kHz}$ USV playback, and this could have led to a state of frustration in the approaching rat. This might also explain why the majority of response calls was emitted within 2 or 3 min after the onset of the $50-\mathrm{kHz}$ USV playback. At first, the animals heard and recognized the stimulus, exhibited a strong social approach immediately afterward and as soon as the rats realized that there was no conspecific present, the emission of response calls increased as an expression of a frustrated state. In line with the frustrated state hypothesis is our finding that the first calls of most animals of data set 2 and 3 were of higher frequencies, i.e., around $50 \mathrm{kHz}$ and quickly changed to calls with frequencies in the 22-kHz USV range (Supplementary Figure 1).

On the other hand, the positive correlation of response calls and approach behavior might serve the hypothesis that the response calls could also be characterized as social contact calls. $50-\mathrm{kHz}$ USV have been postulated to fulfill an affiliative communication function to, for example, maintain a playful state during rough-and-tumble play or as social contact calls to reestablish social proximity after separation of conspecifics (Wöhr et al., 2016). An indication that the response calls in 

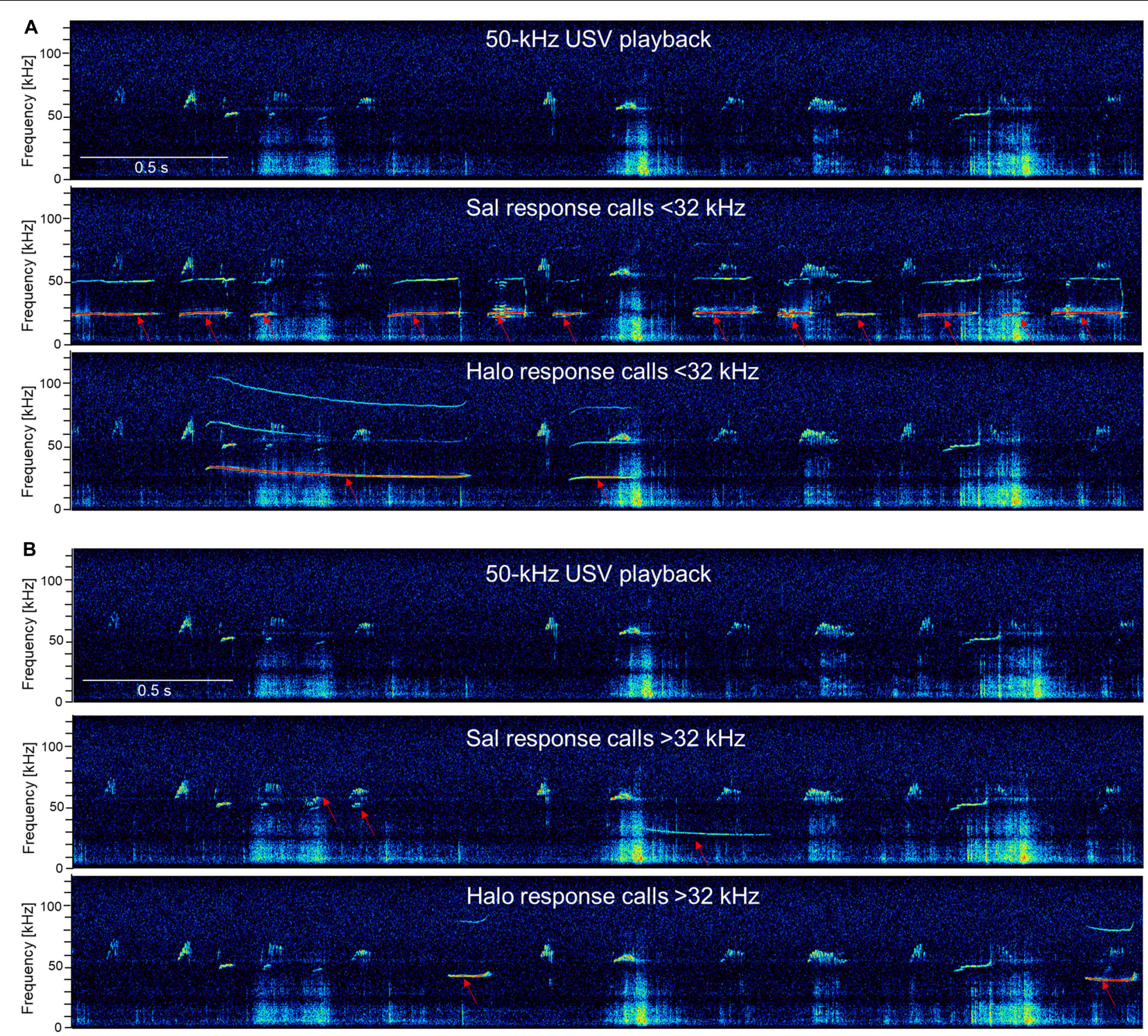

FIGURE 11 | Exemplary response calls during 50-kHz USV playback. The first picture is always the 50-kHz USV playback sequence and the following pictures show response calls in addition to the 50-kHz USV playback sequence (red arrows) $<32 \mathrm{kHz}$ (A) or $>32 \mathrm{kHz}$ (B) of Sal- and Halo-treated rats. Note that the calls depicted for Sal- or Halo-treated rats are not specific to the treatment groups and calls were descriptively similar in all groups.

our study serve as social contact calls is that they are emitted during social approach behavior. Further, such calls are emitted frequently during the approach behavior like $50-\mathrm{kHz}$ USV during rough-and-tumble play (Knutson et al., 1998). In our study we found a moderate positive correlation between response calls and approach behavior, i.e., the time spent close to the active speaker, in SD and, at least to some extent, in WI rats. Apparently, the more the animals tried to reach a possible conspecific signaled by the $50-\mathrm{kHz}$ USV playback, the more calls they emitted, supporting the hypothesis of response calls being contact calls. For Sal- and Halo-treated WI rats, however, this was not the case. In Halo-treated rats, the absence of a positive correlation between approach behavior and response call emission was probably due to the drug-induced immobility (Berz et al., 2021). Since Saltreated rats also received an i.p. injection $60 \mathrm{~min}$ prior to testing, this might have influenced their approach response, as well as their calling behavior; even though Sal-treated rats significantly approached the sound source (Berz et al., 2021) and emitted similar numbers of response calls as WI rats. No correlation was found, however, between overall activity and call numbers in any group. Also or alternatively, the positive correlation between approach behavior and response calls especially observed in SD rats might not be in order to establish contact, but rather due to hypervigilance. Olszyński et al. (2021) showed that in response to $50-\mathrm{kHz}$ USV playback, heart rate and locomotor activity increased as well as the emission of USV. The USV in response 


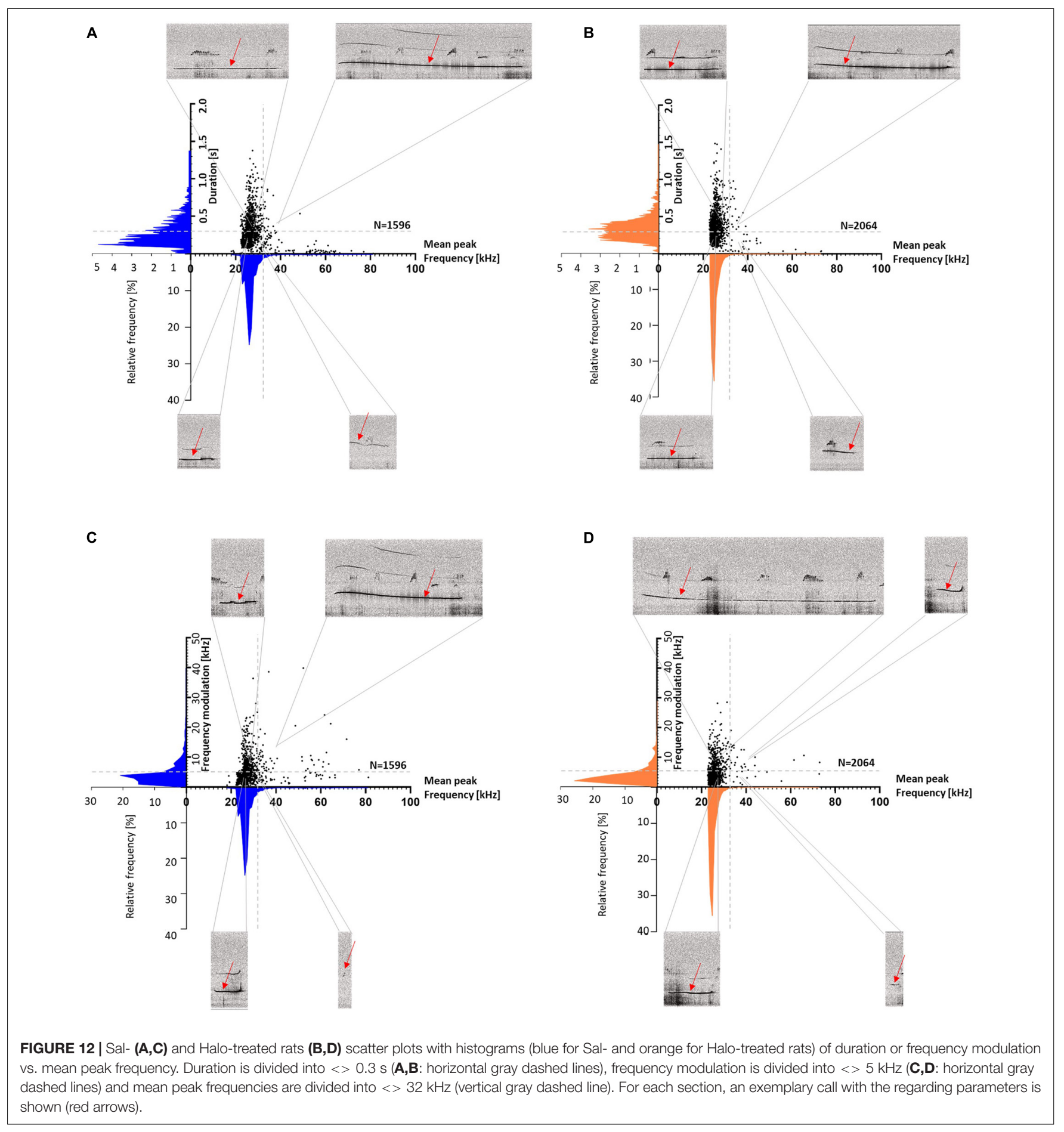

to $50-\mathrm{kHz}$ USV playback in that study were mainly $50-\mathrm{kHz}$ calls, possibly representing contact calls, in contrast to our study here, where the animals mostly emitted calls of lower frequencies. Also, the peak of call emission occurred shortly after the recipient of the playback was in proximity to the sound source and ceased after playback has stopped, which suggests that these calls could function to establish social contact or in search of it. However, the response calls linked to the $50-\mathrm{kHz}$ USV playback do not classify as $50-\mathrm{kHz}$ calls because their mean peak frequencies are much lower, the duration is longer, and there is hardly any frequency modulation compared to $50-\mathrm{kHz}$ calls.

Alternatively, response calls could serve appeasing purposes. The age difference between the rat of the recorded playback and the test subject might be of interest, because in our study, a juvenile rat heard $50-\mathrm{kHz}$ USV playback recorded from an adult rat and accordingly, it seems plausible for the 
TABLE 2 | Scatter plot distributions for Sal- and Halo-treated rats.

\begin{tabular}{|c|c|c|c|c|c|}
\hline \multirow{2}{*}{$\begin{array}{l}\text { Sal } \\
N=15 / 24\end{array}$} & & & \multicolumn{2}{|c|}{ Mean peak frequency } & \multirow[b]{2}{*}{ Total calls } \\
\hline & & & $=32 \mathrm{kHz}$ & $>32 \mathrm{kHz}$ & \\
\hline & \multicolumn{2}{|c|}{ Total numbers (percentages) means \pm SEM } & $1,490(93.4 \%) 72.77 \pm 8.78$ & $106(6.6 \%) 27.23 \pm 8.78$ & $1,596(100 \%)$ \\
\hline \multirow[t]{2}{*}{ Duration } & $<0.3 \mathrm{~s}$ & $1,044(65.4 \%) 59.39 \pm 5.91$ & $960(60.2 \%)$ & $84(5.3 \%)$ & \\
\hline & $>0.3 \mathrm{~s}$ & $552(34.6 \%) 40.61 \pm 5.91$ & $530(33.2 \%)$ & $22(1.4 \%)$ & \\
\hline \multirow[t]{2}{*}{ Modulation } & $<5 \mathrm{kHz}$ & $1,147(71.9 \%) 60.27 \pm 6.73$ & $1,098(68.8 \%)$ & $49(3.1 \%)$ & \\
\hline & $>5 \mathrm{kHz}$ & $449(28.1 \%) 39.73 \pm 6.73$ & $392(24.6 \%)$ & $57(3.6 \%)$ & \\
\hline \multirow{3}{*}{$\begin{array}{l}\text { Halo } \\
N=20 / 24\end{array}$} & & & \multicolumn{2}{|c|}{ Mean peak frequency } & \\
\hline & & & $=32 \mathrm{kHz}$ & $>32 \mathrm{kHz}$ & Total calls \\
\hline & & & $2,021(97.9 \%) 83.44 \pm 6.78$ & $43(2.1 \%) 16.56 \pm 6.78$ & $2,064(100 \%)$ \\
\hline \multirow[t]{2}{*}{ Duration } & $<0.3 \mathrm{~s}$ & $919(44.5 \%) 40.48 \pm 6.89$ & $893(43.3 \%)$ & $26(1.3 \%)$ & \\
\hline & $>0.3 \mathrm{~s}$ & $1,145(55.5 \%) 59.52 \pm 6.89$ & $1,128(54.7 \%)$ & $17(0.8 \%)$ & \\
\hline \multirow[t]{2}{*}{ Modulation } & $<5 \mathrm{kHz}$ & $1,633(79.1 \%) 50.76 \pm 6.73$ & $1,624(78.7 \%)$ & $9(0.4 \%)$ & \\
\hline & $>5 \mathrm{kHz}$ & $431(20.9 \%) 49.24 \pm 6.73$ & 397 (19.2\%) & $34(1.6 \%)$ & \\
\hline
\end{tabular}

Mean peak frequencies $<$ or $>32 \mathrm{kHz}$, Durations $=$ or $>0.3 \mathrm{~s}$, frequency modulations $=$ or $>5 \mathrm{kHz}$.
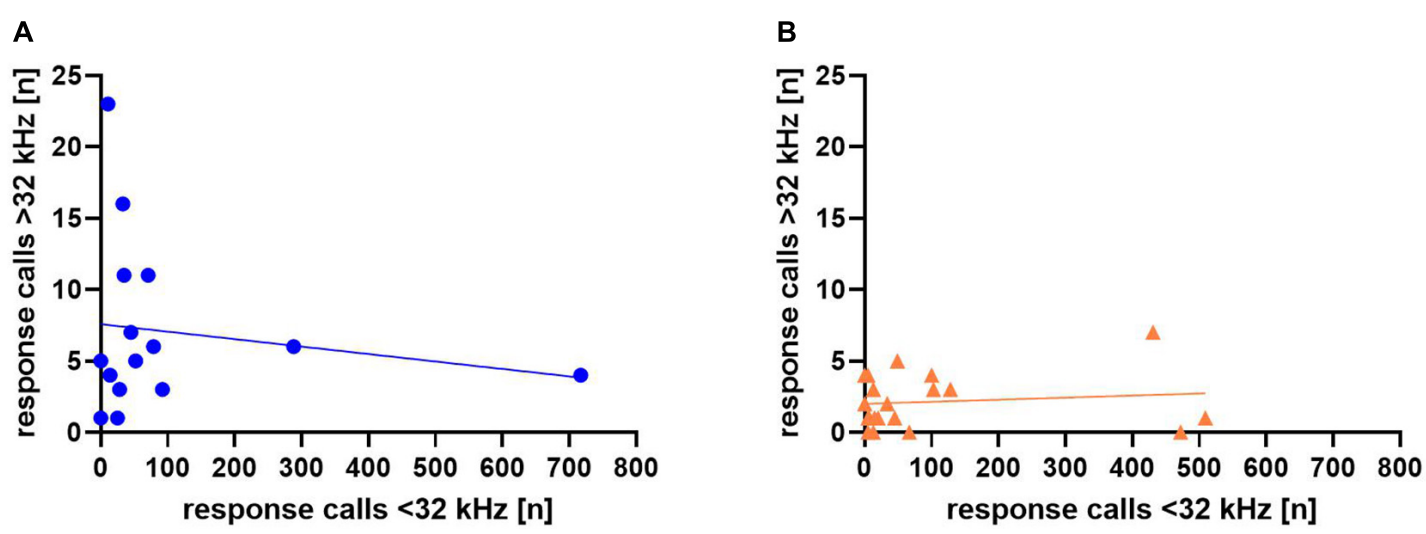

FIGURE 13 | Correlation between calls $<32 \mathrm{kHz}$ and $>32 \mathrm{kHz}$ for Sal- (A) and Halo-treated (B) rats. Each data point represents response calls below and above $32 \mathrm{kHz}$ of one animal.

subject rat to cautiously approach the potential conspecific. Supporting this hypothesis, is the fact that in adult male rats, USV calls of lower frequencies were found during play fighting (Burke et al., 2017, 2020). In social situations that were at risk to escalate into aggression, the play partners lowered their calls gradually from $50 \mathrm{kHz}$ to around $30 \mathrm{kHz}$ with increasing durations (Burke et al., 2017). The authors hypothesized that this group of calls might be a transition from $50-\mathrm{kHz}$ flats to $22-\mathrm{kHz}$ flats or a unique new type of calls. The function of these calls is probably the induction of appeasement, i.e., to de-escalate a situation at risk to turn into aggression (see also Sales, 1972; Lore et al., 1976). Our results seem to support this hypothesis since we tested juvenile rats subjected to calls from an older adult rat and the response calls were in similar frequencies. Moreover, the response calls had also similar frequency modulations, like the calls in the study by Burke et al. (2017) and were not exclusively flat as the common 22-kHz USV. So far, however, it is not known whether receiver rats can gain information about the age of the sender based on their USV.

Importantly, the response call phenomenon studied here in detail appears sufficiently robust to be used as a measure for the reciprocal nature of acoustic communication and can easily be applied in rat model systems for neuropsychiatric disorders, where acoustic communication is impaired, such as autism spectrum disorder (Lai and Baron-Cohen, 2015). In preclinical studies examining USV with the aim to reveal communication deficits in rodent model systems, most laboratories have focused exclusively on the sender. Although there is now an increasing number of preclinical studies including playback paradigms to learn about the responses evoked in the receiver as well (Berg et al., 2018, 2020a,b; Kisko et al., 2018, 2020; Wöhr et al., 2020), 
an important aspect of acoustic communication that is often still neglected is its reciprocal nature and the fact that a signal emitted by the sender frequently evokes the emission of a response signal in the receiver (Seyfarth and Cheney, 2003). Measuring response calls offers a unique opportunity to overcome this limitation. It offers a new approach to studying the reciprocal nature of communication in rodent models for neuropsychiatric disorders.

\section{DATA AVAILABILITY STATEMENT}

The raw data supporting the conclusions of this article will be made available by the authors, without undue reservation.

\section{ETHICS STATEMENT}

The animal study was reviewed and approved by Tierschutzbehoerde, Regierungspraesidium Giessen, Germany, TVA No. 35-2018.

\section{REFERENCES}

Aupperle, R. L., Melrose, A. J., Francisco, A., Paulus, M. P., and Stein, M. B. (2015). Neural substrates of approach-avoidance conflict decision-making. Hum. Brain Mapp. 36, 449-462. doi: 10.1002/HBM.22639

Barker, D. J., Root, D. H., Ma, S., Jha, S., Megehee, L., Pawlak, A. P., et al. (2010). Dose-dependent differences in short ultrasonic vocalizations emitted by rats during cocaine self-administration. Psychopharmacology 211, 435-442. doi: 10.1007/s00213-010-1913-9

Berg, E. L., Ching, T. M., Bruun, D. A., Rivera, J. K., Careaga, M., Ellegood, J., et al. (2020a). Translational outcomes relevant to neurodevelopmental disorders following early life exposure of rats to chlorpyrifos. J. Neurodev. Disord. 12:40. doi: 10.1186/s11689-020-09342-1

Berg, E. L., Pride, M. C., Petkova, S. P., Lee, R. D., Copping, N. A., Shen, Y., et al. (2020b). Translational outcomes in a full gene deletion of ubiquitin protein ligase E3A rat model of Angelman syndrome. Transl. Psychiatry 10:39. doi: 10.1038/s41398-020-0720-2

Berg, E. L., Copping, N. A., Rivera, J. K., Pride, M. C., Careaga, M., Bauman, M. D., et al. (2018). Developmental social communication deficits in the Shank3 rat model of phelan-mcdermid syndrome and autism spectrum disorder. Autism Res. 11, 587-601. doi: 10.1002/aur.1925

Berg, E. L., Jami, S. A., Petkova, S. P., Berz, A., Fenton, T. A., Segal, D. J., et al. (2021). Excessive laughter-like vocalizations, microcephaly, and translational outcomes in the Ube3a deletion rat model of Angelman Syndrome. J. Neurosci. 41, 8801-8814. doi: 10.1523/JNEUROSCI.0925-2 1.2021

Berz, A., Pasquini de Souza, C., Wöhr, M., and Schwarting, R. K. W. (2021). Limited generalizability, pharmacological modulation, and state-dependency of habituation towards pro-social $50-\mathrm{kHz}$ calls in rats. IScience 24:102426. doi: 10.1016/j.isci.2021.102426

Blanchard, R. J., Blanchard, D. C., Agullana, R., and Weiss, S. M. (1991). Twentytwo $\mathrm{kHz}$ alarm cries to presentation of a predator, by laboratory rats living in visible burrow systems. Physiol. Behav. 50, 967-972. doi: 10.1016/00319384(91)90423-L

Brudzynski, S. M. (2001). Pharmacological and behavioral characteristics of $22 \mathrm{kHz}$ alarm calls in rats. Neurosci. Biobehav. Rev. 25, 611-617. doi: 10.1016/S01497634(01)00058-6

Brudzynski, S. M. (2010). Handbook of mammalian vocalization. Handb. Behav. Neurosci. 19, 13-21. doi: 10.1016/B978-0-12-374593-4.00002-4

\section{AUTHOR CONTRIBUTIONS}

RS and MW designed the study, acquired resources and funding, and oversaw the project. $\mathrm{AB}$ performed the experiments. $\mathrm{AB}$ with substantial help from MW analyzed the data. AB, RS, and MW wrote the manuscript. All authors contributed to the article and approved the submitted version.

\section{FUNDING}

This work was supported by grant SCHW 559/15-1 from the Deutsche Forschungsgemeinschaft (DFG) and the DFG funded Research Training Group "Breaking Expectation” (GRK 2271).

\section{SUPPLEMENTARY MATERIAL}

The Supplementary Material for this article can be found online at: https://www.frontiersin.org/articles/10.3389/fnbeh. 2021.812142/full\#supplementary-material

Brudzynski, S. M. (2013a). Ethotransmission: communication of emotional states through ultrasonic vocalization in rats. Curr. Opin. Neurobiol. 23, 310-317. doi: 10.1016/j.conb.2013.01.014

Brudzynski, S. M. (2013b). "Vocalizations as indicators of emotional states in rats and cats," in Evolution of Emotional Communication, eds E. Altenmüller, S. Schmidt, and E. Zimmermann (Oxford: Oxford University Press), 75-91.

Brudzynski, S. M. (2021). Biological functions of rat ultrasonic vocalizations, arousal mechanisms, and call initiation. Brain Sci. 11:605. doi: 10.3390/ brainsci11050605

Brudzynski, S. M., Bihari, F., Ociepa, D., and Fu, X. W. (1993). Analysis of 22 kHz ultrasonic vocalization in laboratory rats: long and short calls. Physiol. Behav. 54, 215-221. doi: 10.1016/0031-9384(93)90102-L

Brudzynski, S. M., and Chiu, E. M. C. (1995). Behavioural responses of laboratory rats to playback of $22 \mathrm{kHz}$ ultrasonic calls. Physiol. Behav. 57, 1039-1044. doi: 10.1016/0031-9384(95)00003-2

Burgdorf, J., Knutson, B., and Panksepp, J. B. (2000). Anticipation of rewarding electrical brain stimulation evokes ultrasonic vocalization in rats. Behav. Neurosci. 114, 320-327. doi: 10.1037/0735-7044.11 4.2.320

Burgdorf, J., Kroes, R. A., Moskal, J. R., Pfaus, J. G., Brudzynski, S. M., and Panksepp, J. B. (2008). Ultrasonic vocalizations of rats (Rattus norvegicus) during mating, play, and aggression: behavioral concomitants, relationship to reward, and self-administration of playback. J. Comp. Psychol. 122, 357-367. doi: $10.1037 / \mathrm{a} 0012889$

Burgdorf, J., Wood, P. L., Kroes, R. A., Moskal, J. R., and Panksepp, J. B. (2007). Neurobiology of $50-\mathrm{kHz}$ ultrasonic vocalizations in rats: electrode mapping, lesion, and pharmacology studies. Behav. Brain Res. 182, 274-283. doi: 10.1016/ j.bbr.2007.03.010

Burke, C. J., Euston, D. R., and Pellis, S. M. (2020). International Journal of Play What do you hear, what do you say? Ultrasonic calls as signals during play fighting in rats. Int. J. Play 9, 92-107. doi: 10.1080/21594937.2020.17 20126

Burke, C. J., Kisko, T. M., Pellis, S. M., and Euston, D. R. (2017). Avoiding escalation from play to aggression in adult male rats: the role of ultrasonic calls. Behav. Process. 144, 72-81. doi: 10.1016/j.beproc.201 7.09.014

Burokas, A., Gutiérrez-Cuesta, J., Martín-García, E., and Maldonado, R. (2012). Operant model of frustrated expected reward in mice. Addict. Biol. 17, 770-782. doi: 10.1111/J.1369-1600.2011.00423.X 
Coffey, K. R., Marx, R. G., and Neumaier, J. F. (2019). DeepSqueak: a deep learning-based system for detection and analysis of ultrasonic vocalizations. Neuropsychopharmacology 44, 859-868. doi: 10.1038/s41386-018-0303-6

Engelhardt, K.-A., Fuchs, E., Schwarting, R. K. W., and Wöhr, M. (2017). Effects of amphetamine on pro-social ultrasonic communication in juvenile rats: implications for mania models. Eur. Neuropsychopharmacol. 27, 261-273. doi: 10.1016/j.euroneuro.2017.01.003

Engelhardt, K. A., Schwarting, R. K. W., and Wöhr, M. (2018). Mapping traitlike socio-affective phenotypes in rats through $50-\mathrm{kHz}$ ultrasonic vocalizations. Psychopharmacology 235, 83-98. doi: 10.1007/s00213-017-4746-y

Fendt, M., Brosch, M., Wernecke, K. E. A., Willadsen, M., and Wöhr, M. (2018). Predator odour but not TMT induces $22-\mathrm{kHz}$ ultrasonic vocalizations in rats that lead to defensive behaviours in conspecifics upon replay. Sci. Rep. 8:11041. doi: 10.1038/s41598-018-28927-4

Kisko, T. M., Braun, M. D., Michels, S., Witt, S. H., Rietschel, M., and Culmsee, C. (2018). Cacnalc haploinsufficiency leads to pro-social $50-\mathrm{kHz}$ ultrasonic communication deficits in rats. Dis. Model Mech. 11:dmm034116. doi: 10.1242/ dmm.034116

Kisko, T. M., Braun, M. D., Michels, S., Witt, S. H., Rietschel, M., Culmsee, C., et al. (2020). Sex-dependent effects of Cacna1c haploinsufficiency on juvenile social play behavior and pro-social $50-\mathrm{kHz}$ ultrasonic communication in rats. Genes Brain Behav. 19:e12552. doi: 10.1111/gbb.12552

Knutson, B., Burgdorf, J., and Panksepp, J. B. (1998). Anticipation of play elicits high-frequency ultrasonic vocalizations in young rats. J. Comp. Psychol. 112, 65-73. doi: 10.1037/0735-7036.112.1.65

Kroes, R. A., Burgdorf, J., Otto, N. J., Panksepp, J., and Moskal, J. R. (2007). Social defeat, a paradigm of depression in rats that elicits $22-\mathrm{kHz}$ vocalizations, preferentially activates the cholinergic signaling pathway in the periaqueductal gray. Behav. Brain Res. 182, 290-300. doi: 10.1016/J.BBR.2007 .03 .022

Lai, M. C., and Baron-Cohen, S. (2015). Identifying the lost generation of adults with autism spectrum conditions. Lancet Psychiatry 2, 1013-1027. doi: 10.1016/ S2215-0366(15)00277-1

Lore, R., Flannelly, K., and Farina, P. (1976). Ultrasounds produced by rats accompany decreases in intraspecific fighting. Aggress. Behav. 2, 175-181.

Ma, S. T., Maier, E. Y., Ahrens, A. M., Schallert, T., and Duvauchelle, C. L. (2010). Repeated intravenous cocaine experience: development and escalation of predrug anticipatory $50-\mathrm{kHz}$ ultrasonic vocalizations in rats. Behav. Brain Res. 212, 109-114. doi: 10.1016/J.BBR.2010.04.001

Manduca, A., Campolongo, P., Palmery, M., Vanderschuren, L. J. M. J., Cuomo, V., and Trezza, V. (2014). Social play behavior, ultrasonic vocalizations and their modulation by morphine and amphetamine in Wistar and Sprague-Dawley rats. Psychopharmacology 231, 1661-1673. doi: 10.1007/s00213-013-3337-9

Olszyński, K. H., Polowy, R., Małż, M., Boguszewski, P. M., and Filipkowski, R. K. (2020). Playback of alarm and appetitive calls differentially impacts vocal, heartrate and motor response in rats. IScience 23:101577. doi: 10.1016/j.isci.2020. 101577

Olszyński, K. H., Polowy, R., Wardak, A. D., Grymanowska, A. W., and Filipkowski, R. K. (2021). Increased vocalization of rats in response to ultrasonic playback as a sign of hypervigilance following fear conditioning. Brain Sci. 11:970.

Panksepp, J. (2005). Affective consciousness: core emotional feelings in animals and humans. Conscious. Cogn. 14, 30-80. doi: 10.1016/J.CONCOG.2004.10.004

Parsana, A. J., Li, N., and Brown, T. H. (2012). Positive and negative ultrasonic social signals elicit opposing firing patterns in rat amygdala. Behav. Brain Res. 226, 77-86. doi: 10.1016/J.BBR.2011.08.040

Rippberger, H., Van Gaalen, M. M., Schwarting, R. K. W., and Wöhr, M. (2015). Environmental and pharmacological modulation of amphetamine- induced 50-kHz ultrasonic vocalizations in rats. Curr. Neuropharmacol. 13, 220-232.

Robakiewicz, I., Polak, M., Rawska, M., Alberski, D., Polowy, R., Wytrychiewicz, K., et al. (2019). Stimulus-seeking in rats is accompanied by increased c-Fos expression in hippocampal CA1 as well as short $22 \mathrm{kHz}$ and flat $50 \mathrm{kHz}$ calls. Acta Neurobiol. Exp. 79, 309-317. doi: 10.21307/ANE2019-029

Sadananda, M., Wöhr, M., and Schwarting, R. K. W. (2008). Playback of 22-kHz and $50-\mathrm{kHz}$ ultrasonic vocalizations induces differential c-fos expression in rat brain. Neurosci. Lett. 435, 17-23. doi: 10.1016/j.neulet.2008.02.002
Sales, G. D. (1972). Ultrasound and aggressive behaviour in rats and other small mammals. Anim. Behav. 20, 88-100. doi: 10.1016/S0003-3472(72) 80177-5

Schwarting, R. K. W. (2018a). Ultrasonic vocalization in female rats: a comparison among three outbred stocks from pups to adults. Physiol. Behav. 196, 59-66. doi: 10.1016/J.PHYSBEH.2018.08.009

Schwarting, R. K. W. (2018b). Ultrasonic vocalization in juvenile and adult male rats: a comparison among stocks. Physiol. Behav. 191, 1-11. doi: 10.1016/J. PHYSBEH.2018.03.023

Scull, J., Davies, K., and Amsel, A. (1970). Behavioral contrast and frustration effect in multiple and mixed fixed-interval schedules in the rat. J. Comp. Physiol. Psychol. 71, 478-483. doi: 10.1037/H002 9160

Seffer, D., Schwarting, R. K. W., and Wöhr, M. (2014). Pro-social ultrasonic communication in rats: insights from playback studies. J. Neurosci. Methods 234, 73-81. doi: 10.1016/j.jneumeth.2014.01.023

Seyfarth, R. M., and Cheney, D. L. (2003). Signalers and receivers in animal communication. Annu. Rev. Psychol. 54, 145-173. doi: 10.1146/annurev.psych. 54.101601.145121

Simmons, S. J., Barker, D. J., and West, M. O. (2018). Ultrasonic vocalizations capture opposing affective states during drug self-administration: revisiting the opponent-process model of addiction. Handb. Behav. Neurosci. 25, 389-399. doi: 10.1016/B978-0-12-809600-0.00037-8

Takahashi, N., Kashino, M., and Hironaka, N. (2010). Structure of rat ultrasonic vocalizations and its relevance to behavior. PLoS One 5:e14115. doi: 10.1371/ journal.pone.0014115

Taylor, J. E., Ficzere, B., St. Louis, J., and Schoenfeld, T. J. (2019). Examining the effects of exercise on frustration-induced anxiety-like behavior in rats. J. Psychol. Res. 24, 210-221. doi: 10.24839/2325-7342.JN2 4.4.210

Tonelli, L. C., Wöhr, M., Schwarting, R. K. W., and Melo-Thomas, L. (2017). Awakenings in rats by ultrasounds: a new animal model for paradoxical kinesia. Behav. Brain Res. 337, 204-209. doi: 10.1016/j.bbr.2017 .09 .021

Willadsen, M., Best, L. M., Wöhr, M., and Clarke, P. B. S. (2018). Effects of anxiogenic drugs on the emission of 22- and $50-\mathrm{kHz}$ ultrasonic vocalizations in adult rats. Psychopharmacology 235, 2435-2445. doi: 10.1007/s00213-0184942-4

Willadsen, M., Seffer, D., Schwarting, R. K. W., and Wöhr, M. (2014). Rodent ultrasonic communication: male prosocial $50-\mathrm{kHz}$ ultrasonic vocalizations elicit social approach behavior in female rats (Rattus norvegicus). J. Comp. Psychol. 128, 56-64. doi: 10.1037/a003 4778

Willuhn, I., Tose, A., Wanat, M. J., Hart, A. S., Hollon, N. G., Phillips, P. E. M., et al. (2014). Phasic dopamine release in the nucleus accumbens in response to pro-social $50 \mathrm{kHz}$ ultrasonic vocalizations in rats. J. Neurosci. 34, 10616-10623. doi: 10.1523/JNEUROSCI.1060-14.2014

Wöhr, M. (2018). Ultrasonic communication in rats: appetitive $50-\mathrm{kHz}$ ultrasonic vocalizations as social contact calls. Behav. Ecol. Sociobiol. 72:14. doi: 10.1007/ s00265-017-2427-9

Wöhr, M. (2021). Measuring mania-like elevated mood through amphetamineinduced $50-\mathrm{kHz}$ ultrasonic vocalizations in rats. Br. J. Pharmacol. [Epub ahead of print]. doi: 10.1111/bph.15487

Wöhr, M., Houx, B., Schwarting, R. K. W., and Spruijt, B. (2008). Effects of experience and context on $50-\mathrm{kHz}$ vocalizations in rats. Physiol. Behav. 93, 766-776. doi: 10.1016/J.PHYSBEH.2007.11.031

Wöhr, M., and Schwarting, R. K. W. (2007). Ultrasonic communication in rats: can playback of $50-\mathrm{kHz}$ calls induce approach behavior? PLoS One 2:e1365. doi: 10.1371/journal.pone.0001365

Wöhr, M., and Schwarting, R. K. W. (2009). Ultrasonic communication in rats: effects of morphine and naloxone on vocal and behavioral responses to playback of 50-kHz vocalizations. Pharmacol. Biochem. Behav. 94, 285-295. doi: 10.1016/ j.pbb.2009.09.008

Wöhr, M., and Schwarting, R. K. (2012). Testing social acoustic memory in rats: effects of stimulus configuration and long-term memory on the induction of social approach behavior by appetitive $50-\mathrm{kHz}$ ultrasonic vocalizations. Neurobiol. Learn. Memory 98, 154-164. 
Wöhr, M., and Schwarting, R. K. W. (2013). Affective communication in rodents: ultrasonic vocalizations as a tool for research on emotion and motivation. Cell Tissue Res. 354, 81-97. doi: 10.1007/s00441-013-1607-9

Wöhr, M., Seffer, D., and Schwarting, R. K. W. (2016). Studying socio-affective communication in rats through playback of ultrasonic vocalizations. Curr. Protoc. Neurosci. 75, 8.35.1-8.35.17.

Wöhr, M., Willadsen, M., Kisko, T. M., Schwarting, R. K. W., and Fendt, M. (2020). Sex-dependent effects of Cacnalc haploinsufficiency on behavioral inhibition evoked by conspecific alarm signals in rats. Prog. Neuro Psychopharmacol. Biol. Psychiatry 99:109849. doi: 10.1016/j.pnpbp.2019.109849

Conflict of Interest: The authors declare that the research was conducted in the absence of any commercial or financial relationships that could be construed as a potential conflict of interest.
The handling editor declared a past co-authorship with one of the authors MW.

Publisher's Note: All claims expressed in this article are solely those of the authors and do not necessarily represent those of their affiliated organizations, or those of the publisher, the editors and the reviewers. Any product that may be evaluated in this article, or claim that may be made by its manufacturer, is not guaranteed or endorsed by the publisher.

Copyright (c) 2022 Berz, Wöhr and Schwarting. This is an open-access article distributed under the terms of the Creative Commons Attribution License (CC BY). The use, distribution or reproduction in other forums is permitted, provided the original author(s) and the copyright owner(s) are credited and that the original publication in this journal is cited, in accordance with accepted academic practice. No use, distribution or reproduction is permitted which does not comply with these terms. 